\title{
Semiflow Selection to Models of General Compressible Viscous Fluids
}

\author{
Danica Basarić(i) \\ Communicated by F. Flandoli
}

\begin{abstract}
We prove the existence of a semiflow selection with range the space of càglàd, i.e. left-continuous and having right-hand limits functions defined on $[0, \infty)$ and taking values in a Hilbert space. Afterwards, we apply this abstract result to the system arising from a compressible viscous fluid with a barotropic pressure of the type $a \varrho^{\gamma}, \gamma \geq 1$, with a viscous stress tensor being a nonlinear function of the symmetric velocity gradient.
\end{abstract}

\section{Introduction}

First developed by Krylov [21] and later adapted by Flandoli and Romito [18], Breit et al. [8] in the context of the Navier-Stokes system, the semiflow selection is an important stochastic tool when studying systems that lack uniqueness: it allows to identify a solution satisfying at least the semigroup property.

Inspired by the deterministic adaptation of Cardona and Kapitanski [11], in the first part of this work we will prove the existence of a semiflow selection in an abstract setting. More precisely, denoting with $H$ a Hilbert space and with $\mathcal{T}=\mathfrak{D}([0, \infty) ; H)$ the Skorokhod space of càglàd functions defined on $[0, \infty)$ and taking values in $H$, we will show the existence of a Borel measurable map $\mathfrak{u}: D \subseteq H \rightarrow \mathcal{T}$ such that for any $x \in D$ and any $t_{1}, t_{2} \geq 0$

$$
\mathfrak{u}(x)\left(t_{1}+t_{2}\right)=\mathfrak{u}\left[\mathfrak{u}(x)\left(t_{1}\right)\right]\left(t_{2}\right) .
$$

One could think that a more natural choice for $\mathcal{T}$ would be the space $C([0, \infty) ; H)$ of continuous functions, as in [11]. However, this option can be too strong: if we want to apply this abstract setting to the typical systems arising from fluid dynamics, where $[0, \infty)$ is the set of times and $\mathcal{T}$ represents the trajectory space, then it is difficult to ensure the energy of the system to be continuous, since it is at most a non-increasing quantity with possible jumps. For the aforementioned reason, in the context of the compressible Euler system, Breit et al. [7,9] considered the energy in the $L^{1}$-space. But the choice $\mathcal{T}=L^{1}([0, \infty) ; H)$ is still not optimal as it is better to work with a space whose elements are well-defined at any point.

In the second part of this work, we will apply the abstract machinery previously achieved to a general model of compressible viscous fluids, described by the following pair of equations:

$$
\begin{aligned}
\partial_{t} \varrho+\operatorname{div}_{x}(\varrho \mathbf{u}) & =0, \\
\partial_{t}(\varrho \mathbf{u})+\operatorname{div}_{x}(\varrho \mathbf{u} \otimes \mathbf{u})+\nabla_{x} p & =\operatorname{div}_{x} \mathbb{S},
\end{aligned}
$$

where the unknown variables are the density $\varrho$ and the velocity $\mathbf{u}$. In this context, the viscous stress tensor $\mathbb{S}$ is connected to the symmetric velocity gradient $\mathbb{D} \mathbf{u}$ through the relation

$$
\mathbb{S}: \mathbb{D} \mathbf{u}=F(\mathbb{D} \mathbf{u})+F^{*}(\mathbb{S})
$$

where $F$ is a proper convex l.s.c. (lower semi-continuous) function and $F^{*}$ denotes its conjugate; moreover, the barotropic pressure will be of the type $p(\varrho)=a \varrho^{\gamma}, \gamma \geq 1$. We will deal with the concept of dissipative solutions, i.e. solutions that satisfy our problem in the weak sense but with an extra defect term in the 
balance of momentum, arising from possible concentrations and/or oscillations in the convective and pressure terms; for more details see Definition 4.1. It is interesting to note that for $\gamma=1$, the defect in the momentum equation vanishes and the latter is satisfied in the sense of distributions. Thus our approach represents an alternative to the "standard" measure-valued framework applied in this context by Matušů-Nečasová and Novotný [23].

Denoting with $\mathcal{P}(\mathcal{T})$ the family of all the subsets of a space $\mathcal{T}$ and introducing the set-valued mapping $\mathcal{U}: D \subseteq H \rightarrow \mathcal{P}(\mathcal{T})$ that associates to every initial data $x \in D$ the family of dissipative solutions $\mathcal{U}(x)$ arising from $x \in D$, the key point in order to get the existence of semiflow selection will be to show that $\mathcal{U}$ satisfies five properties: non-emptiness, compactness, Borel-measurability, shift invariance and continuation.

As we will see, in order to verify the validity of the above mentioned properties, there are two main difficulties we have to overcome: the existence of dissipative solutions and the weak sequential stability of the family of dissipative solutions, arising from a fixed initial data. Luckily, the first problem was recently solved by Abbatiello et al. [2] for $\gamma>1$; following the same strategy, the case $\gamma=1$ can be done as well replacing Lemma 8.1 in [2] with Lemma 5.2 below. Conversely, larger part of this work will be dedicated to solve the second issue.

The present problem, at least for degenerate viscosity, seems closer to the inviscid Euler system rather than its Navier-Stokes (or general non-Newtonian) counterpart. There is a growing piece of evidence, see e.g. the survey paper by Buckmaster and Vicol [10], that uniqueness fails in the class of weak solutions while the (unique) strong solutions blow up in a finite time. The possibility to identify a solution semigroup seems therefore a tempting possibility to save well posedness. Note that this is in a sharp contrast to problems, where uniqueness can be established as a consequence of intrinsic stability, cf. e.g. DiPernaLions theory [13] and its extension to Ambrosio [3], or the theory of viscosity solutions for scalar parabolic equations, see Crandall et al. [12]. The main advantage of this approach is the possibility to identify the class of solutions that maximize the energy dissipation rate. They reflect many important properties with (hypothetical) smooth solutions in the long run: convergence to equilibria and/or certain stable waves, see Feireisl et al. [16], Feireisl and Novotný [17].

The paper is organized as follows.

- In Sect. 2 we will define the Skorokhod space of càglàd functions, constructing a proper metric on it and giving a characterization of convergence.

- In Sect. 3 we will prove the existence of a semiflow selection in the abstract setting, cf. Theorem 3.2.

- In Sect. 4 we will focus on system (1.1), first clarifying the concept of dissipative solution (Sect. 4.1), fixing a proper setting (Sect. 4.2) and finally proving in Sect. 4.3 our main result, i.e., the existence of a semiflow selection, cf. Theorem 4.4.

- Section 5 will be entirely dedicated to prove the weak sequential stability of the solution set of (1.1) for a fixed initial data.

\section{Skorokhod Space}

Given a separable Hilbert space $H$, we define the Skorokhod space $\mathfrak{D}([0, \infty) ; H)$ as the space of the càglàd (an acronym for "continue à gauche, limites à droite") functions defined on $[0, \infty)$ taking values in $H$. More precisely, $\Phi$ belongs to the space $\mathfrak{D}([0, \infty) ; H)$ if it is left-continuous and has right-hand limits:

(i) for $t>0, \Phi(t-)=\lim _{s \uparrow t} \Phi(s)$ exists and $\Phi(t-)=\Phi(t)$;

(ii) for $t \geq 0, \Phi(t+)=\lim _{s \downarrow t} \Phi(s)$ exists.

Unlike in the case of continuous functions, the topology on the space of càglàd functions on an unbounded interval cannot be built up by simply considering functions being càglàd on any compact, see e.g. Jakubowski [20]. Instead, we proceed as follows. 
For every $\Phi \in \mathfrak{D}([0, \infty) ; H)$ we define

$$
\widehat{\Phi}_{T}:= \begin{cases}\Phi(0) & \text { for } t \in[-1,0], \\ \Phi(t) & \text { for } t \in(0, T), \\ \Phi(T) & \text { for } t \in[T, T+1],\end{cases}
$$

and, fixing a basis $\left\{e_{k}\right\}_{k \in \mathbb{N}}$ of the Hilbert space $H$, for every $\Phi, \Psi \in \widehat{\mathfrak{D}}([0, \infty) ; H)$ we define

$$
d_{\infty}(\Phi, \Psi):=\sum_{M=1}^{\infty} \sum_{k=1}^{\infty} \frac{1}{2^{M}} \frac{1}{2^{k}} \frac{d_{M}\left(\left\langle\widehat{\Phi}_{M} ; e_{k}\right\rangle,\left\langle\widehat{\Psi}_{M} ; e_{k}\right\rangle\right)}{1+d_{M}\left(\left\langle\widehat{\Phi}_{M} ; e_{k}\right\rangle,\left\langle\widehat{\Psi}_{M} ; e_{k}\right\rangle\right)},
$$

where $d_{M}$ denotes the Skorokhod metric on the space $\mathfrak{D}([-1, M+1] ; \mathbb{R})$, for all $M \in \mathbb{N}$; for the precise definition of $d_{M}$ and further details on the Skorokhod space we refer to Whitt [25], Chapter 12. It is worth noticing that, even if in [25] the author considered càdlàg ("continue à droite, limites à gauche") functions, the same construction works in our context as well: dealing with the completed graphs of the functions, which are obtained by adding segments joining the left and right limits at each discontinuity point to the graph, the actual value of the function at discontinuity points does not matter, provided that it falls appropriately between the left and right limits.

It is easy to verify that $d_{\infty}$ is a metric on $\mathfrak{D}([0, \infty) ; H)$. Moreover, we have the following result.

Proposition 2.1. Let $\left\{\Phi^{n}\right\}_{n \in \mathbb{N}}$ be a sequence in $\mathfrak{D}([0, \infty) ; H)$. If

$$
\Phi^{n} \underset{n \rightarrow \infty}{\longrightarrow} \Phi \text { in } \mathfrak{D}([0, \infty) ; H)
$$

then for all $k \in \mathbb{N}$

$$
\left\langle\Phi^{n}(t) ; e_{k}\right\rangle \underset{n \rightarrow \infty}{\longrightarrow}\left\langle\Phi(t) ; e_{k}\right\rangle \quad \text { for a.e. } t \in(0, \infty) .
$$

\section{Moreover}

(i) if $\Phi^{n}$ are monotone for all $n \in \mathbb{N}$, conditions (2.3) and (2.4) are equivalent;

(ii) if $\Phi^{n}$ are continuous for all $n \in \mathbb{N}$, condition (2.3) is equivalent to

$$
\sup _{t \in[0, M]}\left|\left\langle\Phi^{n}(t)-\Phi(t) ; e_{k}\right\rangle\right| \underset{n \rightarrow \infty}{\longrightarrow} 0
$$

for all $k, M \in \mathbb{N}$.

Proof. First of all, we will show that (2.3) is equivalent to

$$
\left\langle\widehat{\Phi}_{M} ; e_{k}\right\rangle \underset{n \rightarrow \infty}{\longrightarrow}\left\langle\widehat{\Phi}_{M} ; e_{k}\right\rangle \quad \text { in } \mathfrak{D}([-1, M+1] ; \mathbb{R})
$$

for all $k, M \in \mathbb{N}$. Indeed, if $(2.3)$ holds, let $\varepsilon>0, k, M \in \mathbb{N}$ be fixed and choose a positive $\tilde{\varepsilon}=\tilde{\varepsilon}(\varepsilon)$ such that

$$
\frac{2^{k+M} \tilde{\varepsilon}}{1-2^{k+M} \tilde{\varepsilon}}<\varepsilon
$$

From (2.3), there exists $n_{0}=n_{0}(\tilde{\varepsilon})$ such that

$$
\frac{1}{2^{k+M}} \frac{d_{M}\left(\left\langle\widehat{\Phi}_{M} ; e_{k}\right\rangle,\left\langle\widehat{\Phi}_{M} ; e_{k}\right\rangle\right)}{1+d_{M}\left(\left\langle\widehat{\Phi}_{M}^{n} ; e_{k}\right\rangle,\left\langle\widehat{\Phi}_{M} ; e_{k}\right\rangle\right)} \leq d_{\infty}\left(\Phi^{n}, \Phi\right)<\tilde{\varepsilon}, \quad \text { for all } n \geq n_{0},
$$

which, combined with (2.6), implies

$$
d_{M}\left(\left\langle\widehat{\Phi}_{M} ; e_{k}\right\rangle,\left\langle\widehat{\Phi}_{M} ; e_{k}\right\rangle\right)<\varepsilon \quad \text { for all } n \geq n_{0} .
$$


Vice versa, if (2.5) holds, let $\varepsilon>0$ be fixed and choose $N=N(\varepsilon) \geq 2$, such that $1 / 2^{N}<\varepsilon / 2$. From (2.5), there exists $n_{0}=n_{0}(\varepsilon)$ such that

$$
\max _{k+M \leq N} d_{M}\left(\left\langle\widehat{\Phi}_{M} ; e_{k}\right\rangle,\left\langle\widehat{\Phi}_{M} ; e_{k}\right\rangle\right)<\varepsilon \text { for all } n \geq n_{0} .
$$

For every $n \geq n_{0}$ we obtain

$$
d_{\infty}\left(\Phi^{n}, \Phi\right) \leq \sum_{2 \leq k+M \leq N} \frac{\varepsilon}{2^{k+M}}+\sum_{k+M>N} \frac{1}{2^{k+M}}=\frac{\varepsilon}{2}\left(1-\frac{1}{2^{N-1}}\right)+\frac{1}{2^{N}}<\varepsilon .
$$

Let now (2.5) hold and let $k \in \mathbb{N}$ be fixed. From Theorem 12.5.1. in [25], for each $M \in \mathbb{N}$

$$
\left\langle\widehat{\Phi}_{M}(t) ; e_{k}\right\rangle \underset{n \rightarrow \infty}{\longrightarrow}\left\langle\widehat{\Phi}_{M}(t) ; e_{k}\right\rangle
$$

for all $t$ in a dense subset of $[-1, M+1]$, including -1 and $M+1$,

implying in particular from (2.1) the uniform convergence of $\widehat{\Phi}_{M}$ to $\widehat{\Phi}_{M}$ on $[-1,0]$ and $[M, M+1]$. We then recover that for all $M \in \mathbb{N}$

$$
\left\langle\left.\Phi^{n}\right|_{[0, M]}(t) ; e_{k}\right\rangle \underset{n \rightarrow \infty}{\longrightarrow}\left\langle\left.\Phi\right|_{[0, M]}(t) ; e_{k}\right\rangle
$$

for all $t$ in a dense subset of $[0, M]$. More precisely, denoting with $\operatorname{Disc}_{M}(\Phi)$ the set of discontinuities of $\Phi$ on $[0, M]$, specifically

$$
\operatorname{Disc}_{M}(\Phi):=\{t \in(0, M]: \Phi(t) \neq \Phi(t+)\}
$$

then, by Lemma 12.5.1 in [25], (2.8) holds for all $t \notin D i s c_{M}(\Phi)$. Introducing the set

$$
\operatorname{Disc}(\Phi)=\bigcup_{M \in \mathbb{N}} \operatorname{Disc}_{M}(\Phi)
$$

then trivially $\operatorname{Disc}_{M}(\Phi) \subseteq \operatorname{Disc}_{M+1}(\Phi)$ for all $M \in \mathbb{N}$ and

$$
\left\langle\Phi^{n}(t) ; e_{k}\right\rangle \underset{n \rightarrow \infty}{\longrightarrow}\left\langle\Phi(t) ; e_{k}\right\rangle \quad \text { for all } t \notin \operatorname{Disc}(\Phi) ;
$$

since by Corollary 12.2.1 in [25] each $\operatorname{Disc}_{M}(\Phi)$ is either finite or countable, the set Disc $(\Phi)$ is at most countable and thus we get (2.4).

Furthermore, conditions (i) and (ii) follow easily from the fact that

(i) if $\Phi^{n}$ is monotone for every $n \in \mathbb{N}$ then (2.5) is equivalent to (2.7) for all $k, M \in \mathbb{N}$ by [25], Corollary 12.5.1;

(ii) if $\Phi^{n}$ is continuous for every $n \in \mathbb{N}$ then (2.5) reduces to uniform convergence on the interval $[-1, M+1]$ for all $k, M \in \mathbb{N}$.

\section{Semiflow Selection}

In this section we will focus on proving the existence of a semiflow selection. We start by fixing our setting; from now on, $\mathcal{P}(X)$ will denote the family of all subsets of a space $X$. Let

- $H$ be a separable Hilbert space with a basis $\left\{e_{k}\right\}_{k \in \mathbb{N}}$;

- $D$ a closed convex subset of $H$;

- $\mathcal{T}=\mathfrak{D}([0, \infty) ; H)$;

- $\mathcal{U}: D \rightarrow \mathcal{P}(\mathcal{T})$ satisfy the following properties.

(P1) Non-emptiness: for every $x \in D, \mathcal{U}(x)$ is a non-empty subset of $\mathcal{T}$.

(P2) Compactness: for every $x \in D, \mathcal{U}(x)$ is a compact subset of $\mathcal{T}$.

(P3) Measurability: the map $\mathcal{U}: D \rightarrow \mathcal{P}(\mathcal{T})$ is Borel measurable. 
(P4) Shift invariance: introducing the positive shift operator $S_{T} \circ \Phi$ for every $T>0$ and $\Phi \in \mathcal{T}$ as

$$
S_{T} \circ \Phi(t)=\Phi(T+t), \quad \text { for all } t \geq 0,
$$

then, for any $T>0, x \in D$ and $\Phi \in \mathcal{U}(x)$, we have

$$
S_{T} \circ \Phi \in \mathcal{U}(\Phi(T)) .
$$

(P5) Continuation: introducing the continuation operator $\Phi_{1} \cup_{T} \Phi_{2}$ for every $T>0$ and $\Phi_{1}, \Phi_{2} \in \mathcal{T}$ as

$$
\Phi_{1} \cup_{T} \Phi_{2}(t)=\left\{\begin{array}{ll}
\Phi_{1}(t) & \text { for } 0 \leq t \leq T, \\
\Phi_{2}(t-T) & \text { for } t>T,
\end{array} \text { for all } t \geq 0,\right.
$$

then, for any $T>0, x \in D, \Phi_{1} \in \mathcal{U}(x)$ and $\Phi_{2} \in \mathcal{U}\left(\Phi_{1}(T)\right)$, we have

$$
\Phi_{1} \cup_{T} \Phi_{2} \in \mathcal{U}(x) .
$$

Remark 3.1. It is worth noticing that, in order to guarantee the validity of the shift invariance and continuation properties, it is necessary to verify that for any $x \in D$ and any $\Phi \in \mathcal{U}(x)$, $\Phi$ evaluated at any $t \geq 0$ also belongs to set $D$.

We are now ready to state and prove the following result.

Theorem 3.2. Let the mapping $\mathcal{U}: D \subseteq H \rightarrow \mathcal{P}(\mathcal{T})$ satisfy properties (P1)-(P5) stated above. Then, there exists a Borel measurable map

$$
\mathfrak{u}: D \rightarrow \mathcal{T}, \quad \mathfrak{u}(x) \in \mathcal{U}(x) \text { for every } x \in D,
$$

satisfying the semigroup property: for any $x \in D$ and any $t_{1}, t_{2} \geq 0$

$$
\mathfrak{u}(x)\left(t_{1}+t_{2}\right)=\mathfrak{u}\left[\mathfrak{u}(x)\left(t_{1}\right)\right]\left(t_{2}\right) .
$$

Proof. The idea of the proof is to reduce iteratively the set $\mathcal{U}(x)$ for a fixed $x \in D$, selecting the minimum points of particular functionals in order to obtain finally a single point in $\mathcal{T}$, which will define $\mathfrak{u}(x)$. The procedure has been proposed by Cardona and Kapitanski [11] in the context of continuous trajectories and later adapted to more general setting in [9] and [4].

We introduce the functionals $I_{\lambda, k}: \mathcal{T} \rightarrow \mathbb{R}$ defined for every $\Phi \in \mathcal{T}$ as

$$
I_{\lambda, k}(\Phi)=\int_{0}^{\infty} e^{-\lambda t} f\left(\left\langle\Phi(t) ; e_{k}\right\rangle\right) \mathrm{d} t
$$

where $\lambda>0,\left\{e_{k}\right\}_{k \in \mathbb{N}}$ is a basis in $H$ and $f: \mathbb{R} \rightarrow \mathbb{R}$ is a fixed smooth, bounded and strictly increasing function; this choice is justified by the fact that for a fixed $k \in \mathbb{N}$ we can see $I_{\lambda, k}$ as the Laplace transform of the function $f\left(\left\langle\Phi(\cdot) ; e_{k}\right\rangle\right)$, an useful interpretation for the proof of the existence of the semiflow $\mathfrak{u}$.

Let us first show the continuity of $I_{\lambda, k}$ for every $\lambda>0$ and $k \in \mathbb{N}$ fixed. Let $\left\{\Phi_{n}\right\}_{n \in \mathbb{N}}$ in $\mathcal{T}$ be such that

$$
\Phi_{n} \rightarrow \Phi \quad \text { in } \mathcal{T}
$$

as $n \rightarrow \infty$. By Proposition 2.1, (3.2) implies in particular that

$$
\left\langle\Phi_{n}(t) ; e_{k}\right\rangle \rightarrow\left\langle\Phi(t) ; e_{k}\right\rangle \quad \text { for a.e. } t \in(0, \infty)
$$

as $n \rightarrow \infty$. Due to continuity and boundedness of $f$, we get

$$
I_{\lambda, k}\left(\Phi_{n}\right)=\int_{0}^{\infty} e^{-\lambda t} f\left(\left\langle\Phi_{n}(t) ; e_{k}\right\rangle\right) \mathrm{d} t \rightarrow \int_{0}^{\infty} e^{-\lambda t} f\left(\left\langle\Phi(t) ; e_{k}\right\rangle\right) \mathrm{d} t=I_{\lambda, k}(\Phi),
$$

i.e., what we wanted to prove.

We define the selection mapping for every $x \in D$ as

$$
I_{\lambda, k} \circ \mathcal{U}(x)=\left\{\Phi \in \mathcal{U}(x): I_{\lambda, k}(\Phi) \leq I_{\lambda, k}(\widetilde{\Phi}) \text { for all } \widetilde{\Phi} \in \mathcal{U}(x)\right\}
$$


Notice, in particular, that the minimum exists since $I_{\lambda, k}$ is continuous on $\mathcal{T}$ and the $\operatorname{set} \mathcal{U}(x)$ is compact. From the fact that $\mathcal{U}$ satisfies properties $(\mathbf{P 1})-(\mathbf{P} 5)$, it is not difficult to show that the set-valued mapping

$$
I_{\lambda, k} \circ \mathcal{U}: D \rightarrow \mathcal{P}(\mathcal{T})
$$

satisfies properties (P1)-(P5) as well, cf. Proposition 5.1 in [9].

We are now ready to prove the existence of the semiflow selection $\mathfrak{u}$. Fixing a countable set $\left\{\lambda_{j}\right\}_{j \in \mathbb{N}}$ dense in $(0, \infty)$, we can define the functionals

$$
I_{j, k}(\Phi)=\int_{0}^{\infty} e^{-\lambda_{j} t} f\left(\left\langle\Phi(t) ; e_{k}\right\rangle\right) \mathrm{d} t .
$$

Choosing an enumeration $\{j(i), k(i)\}_{i=1}^{\infty}$ of the all involved combinations of indeces, we define the maps

$$
\mathcal{U}^{i}:=I_{j(i), k(i)} \circ \cdots \circ I_{j(1), k(1)} \circ \mathcal{U}, \quad i=1,2, \ldots,
$$

and

$$
\mathcal{U}^{\infty}:=\bigcap_{i=1}^{\infty} \mathcal{U}^{i}
$$

It is easy to show that the set-valued map

$$
D \ni x \mapsto \mathcal{U}^{\infty}(x) \in \mathcal{P}(\mathcal{T})
$$

satisfies properties (P1)-(P5) as well; for details, see [9], Theorem 2.5 and [4], Theorem 2.2.

We now claim that for every $x \in D$ the set $\mathcal{U}^{\infty}(x)$ is a singleton. Indeed, if $\Phi_{1}, \Phi_{2} \in \mathcal{U}^{\infty}(x)$ for a fixed $x \in D$, then

$$
I_{j(i), k(i)}\left(\Phi_{1}\right)=I_{j(i), k(i)}\left(\Phi_{2}\right)
$$

for all $i=1,2, \ldots$. Since the integrals $I_{j(i), k(i)}$ can be seen as Laplace transforms of the functions $f\left(\left\langle\Phi(\cdot) ; e_{k}\right\rangle\right)$, we can apply Lerch's theorem to deduce that

$$
f\left(\left\langle\Phi_{1}(t) ; e_{k}\right\rangle\right)=f\left(\left\langle\Phi_{2}(t) ; e_{k}\right\rangle\right)
$$

for all $k \in \mathbb{N}$ and a.e. $t \in(0, \infty)$. Since the function $f$ is strictly increasing, we obtain that

$$
\left\langle\Phi_{1}(t) ; e_{k}\right\rangle=\left\langle\Phi_{2}(t) ; e_{k}\right\rangle
$$

for all $k \in \mathbb{N}$ and a.e. $t \in(0, \infty)$; in particular, from $(2.2)$ we get that $d_{\infty}\left(\omega_{1}, \omega_{2}\right)=0$ and thus $\omega_{1}=\omega_{2}$ in $\mathcal{T}$.

Finally, we define the semiflow selection $\mathfrak{u}$ for all $x \in D$ as

$$
\mathfrak{u}(x):=\mathcal{U}^{\infty}(x) \in \mathcal{T}
$$

mesurability follows from the property $(\mathbf{P 3})$ for $\mathcal{U}^{\infty}$, while the semigroup property follows from property (P4): for any $x \in D$ and any $t_{1}, t_{2} \geq 0$

$$
\mathfrak{u}(x)\left(t_{1}+t_{2}\right)=S_{t_{1}} \circ \mathfrak{u}(x)\left(t_{2}\right)=\mathfrak{u}\left(x\left(t_{1}\right)\right)\left(t_{2}\right) .
$$

\section{Semiflow Selection for Compressible Viscous Fluids}

Let us consider a general mathematical model of compressible viscous fluids, represented by the following system

$$
\begin{array}{r}
\partial_{t} \varrho+\operatorname{div}_{x}(\varrho \mathbf{u})=0 \\
\partial_{t}(\varrho \mathbf{u})+\operatorname{div}_{x}(\varrho \mathbf{u} \otimes \mathbf{u})+\nabla_{x} p=\operatorname{div}_{x} \mathbb{S}
\end{array}
$$


here $\varrho=\varrho(t, x)$ denotes the density, $\mathbf{u}=\mathbf{u}(t, x)$ the velocity, $p=p(\varrho)$ the barotropic pressure and $\mathbb{S}$ the viscous stress tensor, which we suppose to be connected to the symmetric velocity gradient

$$
\mathbb{D} \mathbf{u}=\frac{1}{2}\left(\nabla_{x} \mathbf{u}+\nabla_{x}^{T} \mathbf{u}\right)
$$

through the Fenchel's identity

$$
\mathbb{S}: \mathbb{D} \mathbf{u}=F(\mathbb{D} \mathbf{u})+F^{*}(\mathbb{S}),
$$

where, denoting with $\mathbb{R}_{s y m}^{d \times d}$ the space of $d$-dimensional real symmetric tensors,

$$
F: \mathbb{R}_{s y m}^{d \times d} \rightarrow[0, \infty) \text { is a convex l.s.c. function with } F(0)=0,
$$

and $F^{*}$ is its conjugate, defined for every $\mathbb{A} \in \mathbb{R}_{\text {sym }}^{d \times d}$ as

$$
F^{*}(\mathbb{A}):=\sup _{\mathbb{B} \in \mathbb{R}_{s y m}^{d \times d}}\{\mathbb{A}: \mathbb{B}-F(\mathbb{B})\} .
$$

Notice that conditions (4.4) guarantees that

$$
F^{*}: \mathbb{R}_{\mathrm{sym}}^{d \times d} \rightarrow[0, \infty] \text { is a superlinear l.s.c. function, } \quad \liminf _{|\mathbb{S}| \rightarrow \infty} \frac{F^{*}(\mathbb{S})}{|\mathbb{S}|}=\infty .
$$

Furthermore, we will suppose $F$ to satisfy relation

$$
F(\mathbb{D}) \geq \mu\left|\mathbb{D}-\frac{1}{d} \operatorname{Tr}[\mathbb{D}] \mathbb{I}\right|^{q} \quad \text { for all }|\mathbb{D}|>1,
$$

for some $\mu>0$ and $q>1$. Notice that condition (4.3) is equivalent in requiring

$$
\mathbb{S} \in \partial F(\mathbb{D} \mathbf{u}),
$$

where $\partial$ denotes the subdifferential of a convex function.

Regarding pressure, we will consider the standard isentropic case

$$
p(\varrho)=a \varrho^{\gamma}, \quad \gamma \geq 1,
$$

with $a$ a positive constant; however, more general EOS preserving the essential features of (4.7) can be considered, cf. [2]. The pressure potential $P$, satisfying the ODE

$$
\varrho P^{\prime}(\varrho)-P(\varrho)=p(\varrho),
$$

will be of the form

$$
P(\varrho)= \begin{cases}a \varrho \log \varrho & \text { if } \gamma=1 \\ \frac{a}{\gamma-1} \varrho^{\gamma} & \text { if } \gamma>1\end{cases}
$$

in particular, this implies that

$$
P \text { is a strictly convex superlinear continuous function on }[0, \infty) \text {. }
$$

We will study the system on the set $(0, \infty) \times \Omega$, where the physical domain $\Omega \subset \mathbb{R}^{d}$ is assumed to be bounded and Lipschitz, on the boundary of which we impose the no-slip condition

$$
\left.\mathbf{u}\right|_{\partial \Omega}=0 .
$$

Finally, we fix the initial conditions

$$
\varrho(0, \cdot)=\varrho_{0}, \quad(\varrho \mathbf{u})(0, \cdot)=\mathbf{m}_{0} .
$$

Our goal is to apply the abstract machinery introduced in the previous section in order to show the existence of a semiflow selection for system (4.1)-(4.11). More precisely, we aim to prove Theorem 4.4 below, clarifying first the concept of solution we will work with and fixing a proper setting. 


\subsection{Dissipative Solution}

As already mentioned in the introduction, inspired by the recent work of Abbatiello, Feireisl and Novotný [2], we will refer to the concept of dissipative solutions. From now on, it is better to consider the density $\varrho$ and the momentum $\mathbf{m}=\varrho \mathbf{u}$ as state variables, since they are at least weakly continuous in time.

Definition 4.1. The pair of functions $[\varrho, \mathbf{m}]$ constitutes a dissipative solution to the problem (4.1)-(4.11) with the total energy $E$ and initial data

$$
\left[\varrho_{0}, \mathbf{m}_{0}, E_{0}\right] \in L^{\gamma}(\Omega) \times L^{\frac{2 \gamma}{\gamma+1}}\left(\Omega ; \mathbb{R}^{d}\right) \times[0, \infty)
$$

if the following holds:

(i) $\varrho \geq 0$ in $(0, \infty) \times \Omega$ and

$$
[\varrho, \mathbf{m}, E] \in C_{\text {weak }, \text { loc }}\left([0, \infty) ; L^{\gamma}(\Omega)\right) \times C_{\text {weak }, \text { loc }}\left([0, \infty) ; L^{\frac{2 \gamma}{\gamma+1}}\left(\Omega ; \mathbb{R}^{d}\right)\right) \times \mathfrak{D}([0, \infty)) ;
$$

(ii) the integral identity

$$
\left[\int_{\Omega} \varrho \varphi(t, \cdot) \mathrm{d} x\right]_{t=0}^{t=\tau}=\int_{0}^{\tau} \int_{\Omega}\left[\varrho \partial_{t} \varphi+\mathbf{m} \cdot \nabla_{x} \varphi\right] \mathrm{d} x \mathrm{~d} t
$$

holds for any $\tau>0$ and any $\varphi \in C_{c}^{1}([0, \infty) \times \bar{\Omega})$, with $\varrho(0, \cdot)=\varrho_{0}$;

(iii) there exist

$$
\mathbb{S} \in L_{\text {loc }}^{1}\left(0, \infty ; L^{1}\left(\Omega ; \mathbb{R}_{\text {sym }}^{d \times d}\right)\right) \quad \text { and } \quad \Re \in L_{\text {weak }}^{\infty}\left(0, \infty ; \mathcal{M}^{+}\left(\bar{\Omega} ; \mathbb{R}_{\text {sym }}^{d \times d}\right)\right)
$$

such that the integral identity

$$
\begin{aligned}
{\left[\int_{\Omega} \mathbf{m} \cdot \boldsymbol{\varphi}(t, \cdot) \mathrm{d} x\right]_{t=0}^{t=\tau} } & =\int_{0}^{\tau} \int_{\Omega}\left[\mathbf{m} \cdot \partial_{t} \boldsymbol{\varphi}+\mathbb{1}_{\varrho>0} \frac{\mathbf{m} \otimes \mathbf{m}}{\varrho}: \nabla_{x} \boldsymbol{\varphi}+p(\varrho) \operatorname{div}_{x} \boldsymbol{\varphi}\right] \mathrm{d} x \mathrm{~d} t \\
& -\int_{0}^{\tau} \int_{\Omega} \mathbb{S}: \nabla_{x} \boldsymbol{\varphi} \mathrm{d} x \mathrm{~d} t+\int_{0}^{\tau} \int_{\bar{\Omega}} \nabla_{x} \boldsymbol{\varphi}: \mathrm{d} \mathfrak{R} \mathrm{d} t
\end{aligned}
$$

holds for any $\tau>0$ and any $\varphi \in C_{c}^{1}\left([0, \infty) \times \bar{\Omega} ; \mathbb{R}^{d}\right),\left.\varphi\right|_{\partial \Omega}=0$, with $\mathbf{m}(0, \cdot)=\mathbf{m}_{0}$;

(iv) there exists

$$
\mathbf{u} \in L_{\text {loc }}^{q}\left(0, \infty ; W_{0}^{1, q}\left(\Omega ; \mathbb{R}^{d}\right)\right) \text { such that } \mathbf{m}=\varrho \mathbf{u} \text { a.e. in }(0, \infty) \times \Omega
$$

(v) there exist a constant $\lambda>0$ and a càglàd function $E$, non-increasing in $[0, \infty)$, satisfying

$$
\int_{\Omega}\left[\frac{1}{2} \frac{|\mathbf{m}|^{2}}{\varrho}+P(\varrho)\right](\tau, \cdot) \mathrm{d} x+\frac{1}{\lambda} \int_{\bar{\Omega}} \mathrm{d} \operatorname{Tr}[\Re(\tau)]=\mathrm{E}(\tau)
$$

for a.e. $\tau>0$, such that the energy inequality

$$
[E(t) \psi(t)]_{t=\tau_{1}^{-}}^{t=\tau^{+}}-\int_{\tau_{1}}^{\tau_{2}} E \psi^{\prime} \mathrm{d} t+\int_{\tau_{1}}^{\tau_{2}} \psi \int_{\Omega}\left[F(\mathbb{D} \mathbf{u})+F^{*}(\mathbb{S})\right] \mathrm{d} x \mathrm{~d} t \leq 0
$$

holds for any $0 \leq \tau_{1} \leq \tau_{2}$ and any $\psi \in C_{c}^{1}[0, \infty), \psi \geq 0$, with $E(0-)=E_{0} \geq E(0+)$.

Remark 4.2. In this context, $\mathcal{M}^{+}(\bar{\Omega})$ represents the space of all the positive Borel measures on $\bar{\Omega}$, while $\mathcal{M}^{+}\left(\bar{\Omega} ; \mathbb{R}_{\text {sym }}^{d \times d}\right)$ denotes the space of tensor-valued (signed) Borel measures $\mathfrak{R}$ such that

$$
\mathfrak{R}:(\xi \otimes \xi) \in \mathcal{M}^{+}(\bar{\Omega}),
$$

for all $\xi \in \mathbb{R}^{d}$, and with components $\mathfrak{R}_{i, j}=\mathfrak{R}_{j, i} . L_{\text {weak }}^{\infty}(0, \infty ; \mathcal{M}(\bar{\Omega}))$ denotes the space of all the weak-* measurable mapping $\nu:[0, \infty) \rightarrow \mathcal{M}(\bar{\Omega})$ such that

$$
\operatorname{ess}_{\sup } \sin \|\nu(t, \cdot)\|_{\mathcal{M}(\bar{\Omega})}<\infty,
$$

which can also be identified as the dual space of $L^{1}(0, \infty ; C(\bar{\Omega}))$. 
Remark 4.3. The notion of solution introduced in Definition 4.1 satisfies the weak-strong uniqueness principle: a dissipative solution of (4.1)-(4.11) coincides with the strong solution of the same problem emanating from the same initial data as long as the latter exists, cf. Theorem 6.3 in [2].

4.1.1. Short Remark on the Reynolds Stress. The concentration measure $\mathfrak{R}$, that we may call Reynolds stress, appearing in the weak formulation of the balance of momentum (4.13) arises from possible oscillations and/or concentrations in the convective and pressure terms

$$
\mathbb{1}_{\varrho>0} \frac{\mathbf{m} \otimes \mathbf{m}}{\varrho}+p(\varrho) \mathbb{I}
$$

when $\gamma>1$, while for $\gamma=1$, i.e. when the pressure is a linear function of the density $\varrho$, it is only the convective term that contributes to $\mathfrak{R}$.

By consistency, as clearly explained in [2], we should have introduced the dissipation defect $\mathfrak{E} \in$ $L_{\text {weak }}^{\infty}\left(0, \infty ; \mathcal{M}^{+}(\bar{\Omega})\right)$ of the total energy arising from possible concentrations and/or oscillations in the kinetic and potential energy terms

$$
\frac{1}{2} \frac{|\mathbf{m}|^{2}}{\varrho}+P(\varrho)
$$

Instead of (4.14) we would then have

$$
E(\tau)=\int_{\Omega}\left[\frac{1}{2} \frac{|\mathbf{m}|^{2}}{\varrho}+P(\varrho)\right](\tau, \cdot) \mathrm{d} x+\int_{\bar{\Omega}} \mathrm{d} \mathfrak{E}(\tau),
$$

satisfying the energy inequality (4.15). Choosing a positive constant $\lambda>0$ such that

$$
\operatorname{Tr}\left[\mathbb{1}_{\varrho>0} \frac{\mathbf{m} \otimes \mathbf{m}}{\varrho}+\chi(\gamma) p(\varrho) \mathbb{I}\right]=\frac{|\mathbf{m}|^{2}}{\varrho}+d(\gamma-1) P(\varrho) \leq \lambda\left(\frac{1}{2} \frac{|\mathbf{m}|^{2}}{\varrho}+P(\varrho)\right)
$$

with

$$
\chi(\gamma)= \begin{cases}0 & \text { if } \gamma=1 \\ 1 & \text { if } \gamma>1\end{cases}
$$

and adapting Lemma 1.6 in [5], we could recover the compatibility condition

$$
\frac{1}{\lambda} \operatorname{Tr}[\mathfrak{R}(\tau)] \leq \mathfrak{E}(\tau) \quad \text { for a.e. } \tau>0 .
$$

In this sense, our choice of the energy (4.14) makes the problem more general and easier to handle with only one free quantity instead of two; however, it reduces to (4.16) simply choosing a dissipation defect $\mathfrak{E}$ of the type

$$
\mathfrak{E}(\tau):=\frac{1}{\lambda} \operatorname{Tr}[\mathfrak{R}(\tau)] \text { for a.e. } \tau>0 .
$$

\subsection{Set-Up}

First of all, we must fix the space $H$, the subset $D \subseteq H$ and the map $\mathcal{U}$ introduced at the beginning of Sect. 3. In this context

- $H:=W^{-k, 2}(\Omega) \times W^{-k, 2}\left(\Omega ; \mathbb{R}^{d}\right) \times \mathbb{R}$, where the natural number $k>\frac{d}{2}+1$ is fixed;

- $D$ represents the space of initial data; it can be chosen as

$$
D:=\left\{\left[\varrho_{0}, \mathbf{m}_{0}, E_{0}\right] \in H: \varrho_{0} \in L^{1}(\Omega), \varrho_{0} \geq 0, \mathbf{m}_{0} \in L^{1}\left(\Omega ; \mathbb{R}^{d}\right) \text { satisfying }(4.18)\right\}
$$

where

$$
\int_{\Omega}\left[\frac{1}{2} \frac{\left|\mathbf{m}_{0}\right|^{2}}{\varrho_{0}}+P\left(\varrho_{0}\right)\right] \mathrm{d} x \leq E_{0}
$$

- $\mathcal{T}=\mathfrak{D}([0, \infty) ; H)$ represents the trajectory space; 
- $\mathcal{U}: D \rightarrow \mathcal{P}(\mathcal{T})$ represents the set-valued mapping that associate to every $\left[\varrho_{0}, \mathbf{m}_{0}, E_{0}\right] \in D$ the family of dissipative solutions in the sense of Definition 4.1 arising from the initial data $\left[\varrho_{0}, \mathbf{m}_{0}, E_{0}\right]$. More precisely, for every $\left[\varrho_{0}, \mathbf{m}_{0}, E_{0}\right] \in D$

$$
\begin{aligned}
& \mathcal{U}\left[\varrho_{0}, \mathbf{m}_{0}, E_{0}\right]= \\
& \quad\left\{[\varrho, \mathbf{m}, E] \in \mathcal{T}:[\varrho, \mathbf{m}, E] \text { is a dissipative solution with initial data }\left[\varrho_{0}, \mathbf{m}_{0}, E_{0}\right]\right\} .
\end{aligned}
$$

Notice that everything is well-defined; indeed, denoting with $L_{+}^{1}(\Omega)$ the space of non-negative integrable functions on $\Omega$, we can rewrite $D$ as

$$
\left\{\left[\varrho_{0}, \mathbf{m}_{0}, E_{0}\right] \in L_{+}^{1}(\Omega) \times L^{1}\left(\Omega ; \mathbb{R}^{d}\right) \times \mathbb{R}: g\left(\varrho_{0}, \mathbf{m}_{0}\right) \leq E_{0}\right\},
$$

so that it coincides with the epigraph of the function $g: L_{+}^{1}(\Omega) \times L^{1}\left(\Omega ; \mathbb{R}^{d}\right) \rightarrow[0,+\infty]$ defined as

$$
g\left(\varrho_{0}, \mathbf{m}_{0}\right)=\int_{\Omega}\left[\frac{1}{2} \frac{\left|\mathbf{m}_{0}\right|^{2}}{\varrho_{0}}+P\left(\varrho_{0}\right)\right] d x .
$$

From (4.9) and the fact that

$$
[\varrho, \mathbf{m}] \mapsto \begin{cases}0 & \text { if } \mathbf{m}=0 \\ \frac{|\mathbf{m}|^{2}}{\varrho} & \text { if } \varrho>0 \\ \infty & \text { otherwise }\end{cases}
$$

we get that the function $g$ is lower semi-continuous and convex and thus its epigraph is a closed convex subset of $L^{\gamma}(\Omega) \times L^{\frac{2 \gamma}{\gamma+1}}\left(\Omega ; \mathbb{R}^{d}\right) \times \mathbb{R}$ for all $\gamma \geq 1$.

From our choice of $k$, we can use the Sobolev embedding

$$
L^{r}(\Omega) \hookrightarrow \hookrightarrow W^{-k, 2}(\Omega) \quad \text { for every } r \geq 1
$$

to conclude that

$$
C_{\text {weak }, \mathrm{loc}}\left([0, \infty) ; L^{r}(\Omega)\right) \hookrightarrow C_{\mathrm{loc}}\left([0, \infty) ; W^{-k, 2}(\Omega)\right) \hookrightarrow \mathfrak{D}\left([0, \infty) ; W^{-k, 2}(\Omega)\right),
$$

for every $r \geq 1$. Furthermore, due to the weak continuity of the density $\varrho$ and the momentum $\mathbf{m}$, for every fixed $T>0$ and every $t \in[0, T]$, from the energy inequality we can deduce that

$$
\begin{aligned}
& \|\varrho(t, \cdot)\|_{L^{\gamma}(\Omega)} \leq \sup _{t \in[0, T]}\|\varrho(t, \cdot)\|_{L^{\gamma}(\Omega)} \leq c \sup _{t \in[0, T]}\|1+P(\varrho)(t, \cdot)\|_{L^{1}(\Omega)} \leq c\left(E_{0}, \Omega\right), \\
& \|\mathbf{m}(t, \cdot)\|_{L^{\frac{2 \gamma}{\gamma+1}}\left(\Omega ; \mathbb{R}^{d}\right)} \leq \sup _{t \in[0, T]}\|\mathbf{m}(t, \cdot)\|_{L^{\frac{2 \gamma}{\gamma+1}}\left(\Omega ; \mathbb{R}^{d}\right)} \\
& \leq \operatorname{ess} \sup _{t \in(0, T)}\left\|\frac{\mathbf{m}}{\sqrt{\varrho}}(t, \cdot)\right\|_{L^{2}\left(\Omega ; \mathbb{R}^{d}\right)}\|\sqrt{\varrho}(t, \cdot)\|_{L^{2 \gamma}(\Omega)} \leq c\left(E_{0}, \Omega\right) ;
\end{aligned}
$$

Finally, from condition (i) of Definition 4.1 we also have that $\varrho(t, \cdot) \geq 0$ for all $t \geq 0$, while relation

$$
\int_{\Omega}\left[\frac{1}{2} \frac{|\mathbf{m}|^{2}}{\varrho}+P(\varrho)\right](t, \cdot) d x \leq E(t-)=E(t)
$$

holds for all $t \geq 0$ since the energy is convex and $\varrho$ and $\mathbf{m}$ are weakly continuous in time. In particular, we have that for every $t \geq 0$

$$
[\varrho(t, \cdot), \mathbf{m}(t, \cdot), E(t)] \in D .
$$




\subsection{Main Result}

Keeping in mind the notation introduced in the previous section, we are now ready to state our main result.

Theorem 4.4. System (4.1)-(4.11) admits a semiflow selection $U$ in the class of dissipative solutions, i.e., there exists a Borel measurable map $U: D \rightarrow \mathcal{T}$ such that

$$
U\left[\varrho_{0}, \boldsymbol{m}_{0}, E_{0}\right] \in \mathcal{U}\left[\varrho_{0}, \boldsymbol{m}_{0}, E_{0}\right] \text { for every }\left[\varrho_{0}, \boldsymbol{m}_{0}, E_{0}\right] \in D
$$

satisfying the semigroup property: for any $\left[\varrho_{0}, \boldsymbol{m}_{0}, E_{0}\right] \in D$ and any $t_{1}, t_{2} \geq 0$

$$
U\left[\varrho_{0}, \boldsymbol{m}_{0}, E_{0}\right]\left(t_{1}+t_{2}\right)=U\left[\varrho\left(t_{1}\right), \boldsymbol{m}\left(t_{1}\right), E\left(t_{1}\right)\right]\left(t_{2}\right)
$$

where $[\varrho, m, E]=U\left[\varrho_{0}, \boldsymbol{m}_{0}, E_{0}\right]$.

Theorem 4.4 is a consequence of Theorem 3.2 once we have verified that $\mathcal{U}$ satisfies properties $(\mathbf{P} \mathbf{1})-$ (P5). To this end, we emphasise the following points.

- Property (P1) is equivalent in showing the existence of a dissipative solution in the sense of Definition 4.1 for any fixed initial data $\left[\varrho_{0}, \mathbf{m}_{0}, E_{0}\right] \in D$. For $\gamma>1$, this is the main result achieved in [2], Sect. 3, while the case $\gamma=1$ can be done as well applying Lemma 5.2 below instead of Lemma 8.1 in $[2]$.

- Properties (P2) and (P3) hold true if we manage to prove the weak sequential stability of the solution set $\mathcal{U}\left[\varrho_{0}, \mathbf{m}_{0}, E_{0}\right]$ for every $\left[\varrho_{0}, \mathbf{m}_{0}, E_{0}\right] \in D$ fixed, since it will in particular imply compactness and the closed-graph property of the mapping

$$
D \ni\left[\varrho_{0}, \mathbf{m}_{0}, E_{0}\right] \rightarrow \mathcal{U}\left[\varrho_{0}, \mathbf{m}_{0}, E_{0}\right] \in \mathcal{P}(\mathcal{T})
$$

and thus the Borel-measurality of $\mathcal{U}$, cf. Lemma 12.1.8 in [24].

- Properties (P4) and (P5) can be easily checked following the same arguments done in [9], Lemma 4.2 and 4.3 .

In conclusion, we are done if we show the weak sequential stability of the solution set $\mathcal{U}\left[\varrho_{0}, \mathbf{m}_{0}, E_{0}\right]$ for every $\left[\varrho_{0}, \mathbf{m}_{0}, E_{0}\right] \in D$ fixed. Being the proof quite elaborated, it is postponed to the next section.

Remark 4.5. As already done for the Euler and Navier-Stokes systems, cf. [4,9], among all the dissipative solutions emanating from the same initial data it is possible to select only the admissible ones, i.e., satisfying the physical principal of minimizing the total energy or equivalently, that are minimal with respect to relation $\prec$ defined as

$$
\left[\varrho^{1}, \mathbf{m}^{1}, E^{1}\right] \prec\left[\varrho^{2}, \mathbf{m}^{2}, E^{2}\right] \quad \Leftrightarrow \quad E^{1}(\tau \pm) \leq E^{2}(\tau \pm) \text { for any } \tau \in(0, \infty),
$$

where $\left[\varrho^{i}, \mathbf{m}^{i}, E^{i}\right], i=1,2$ are two dissipative solutions sharing the same initial data. Indeed, it is sufficient to start the selection considering in (3.1) the functional $I_{1, k}$ with the function $f$ such that

$$
f\left(\left\langle\Phi(t) ; e_{k}\right\rangle\right)=f(E(t)) \text { for all } t \geq 0,
$$

where $\Phi(t)=[\varrho(t), \mathbf{m}(t), E(t)]$; see [9], Lemma 5.2 for more details.

\section{Weak Sequential Stability}

This section will be entirely dedicated to the proof of the following result.

Proposition 5.1. Let $\left\{\left[\varrho_{n}, \boldsymbol{m}_{n}\right]\right\}_{n \in \mathbb{N}}$ be a family of dissipative solutions with the corresponding total energies $\left\{E_{n}\right\}_{n \in \mathbb{N}}$ and initial data $\left\{\left[\varrho_{0, n}, \boldsymbol{m}_{0, n}, E_{0, n}\right]\right\}_{n \in \mathbb{N}}$ in the sense of Definition 4.1. If

$$
\left[\varrho_{0, n}, \boldsymbol{m}_{0, n}, E_{0, n}\right] \rightarrow\left[\varrho_{0}, \boldsymbol{m}_{0}, E_{0}\right] \quad \text { in } H,
$$

then, at least for suitable subsequences,

$$
\left[\varrho_{n}, \boldsymbol{m}_{n}, E_{n}\right] \rightarrow[\varrho, \boldsymbol{m}, E] \quad \text { in } \mathfrak{D}([0, \infty) ; H)
$$


where $H=W^{-k, 2}(\Omega) \times W^{-k, 2}\left(\Omega ; \mathbb{R}^{d}\right) \times \mathbb{R}$ with the natural number $k>\frac{d}{2}+1$ fixed, and $[\varrho, \boldsymbol{m}]$ is another dissipative solution of the same problem with total energy $E$.

The proof will be divided in four steps:

1. in Sect. 5.1 we will first deduce a family of uniform bounds and convergences, including the limits $\varrho$ of the densities, $\mathbf{m}$ of the momenta and $\mathbf{u}$ of the velocities;

2. in Sect. 5.2 we will pass to the limit in the weak formulation of the continuity equation and the balance of momentum;

3. in order to show that $\mathbf{m}$ can be written as the product $\varrho \mathbf{u}$, in Sect. 5.3 we will state and prove Lemma 5.2;

4. finally, in Sect. 5.4 we will focus on finding the limit $E$ of the energies.

\subsection{Uniform Bounds and Limits Establishment}

Our first goal is to show the following convergences, passing to suitable subsequences as the case may be:

$$
\begin{aligned}
& \varrho_{n} \rightarrow \varrho \quad \text { in } C_{\text {weak }, \text { loc }}\left([0, \infty) ; L^{\gamma}(\Omega)\right), \\
& \mathbf{m}_{n} \rightarrow \mathbf{m} \quad \text { in } C_{\text {weak }, \text { loc }}\left([0, \infty) ; L^{\frac{2 \gamma}{\gamma+1}}\left(\Omega ; \mathbb{R}^{d}\right)\right) \text {, } \\
& \mathbf{u}_{n} \rightarrow \mathbf{u} \text { in } L_{\mathrm{loc}}^{q}\left(0, \infty ; W_{0}^{1, q}\left(\Omega ; \mathbb{R}^{d}\right)\right) \\
& \mathbb{S}_{n} \rightarrow \mathbb{S} \quad \text { in } L_{\text {loc }}^{1}\left(0, \infty ; L^{1}\left(\Omega ; \mathbb{R}^{d \times d}\right)\right), \\
& \mathbb{1}_{\varrho_{n}>0} \frac{\mathbf{m}_{n} \otimes \mathbf{m}_{n}}{\varrho_{n}} \stackrel{*}{\rightarrow} \overline{\mathbb{1}_{\varrho>0} \frac{\mathbf{m} \otimes \mathbf{m}}{\varrho}} \text { in } L_{\text {weak }}^{\infty}\left(0, \infty ; \mathcal{M}\left(\bar{\Omega} ; \mathbb{R}_{\mathrm{sym}}^{d \times d}\right)\right) \text {, } \\
& p\left(\varrho_{n}\right) \stackrel{*}{\rightarrow} \overline{p(\varrho)} \quad \text { in } L_{\mathrm{weak}}^{\infty}(0, \infty ; \mathcal{M}(\bar{\Omega})) \quad \text { if } \gamma>1, \\
& \mathfrak{R}_{n} \stackrel{*}{\rightarrow} \widetilde{\Re} \quad \text { in } L_{\text {weak }}^{\infty}\left(0, \infty ; \mathcal{M}^{+}\left(\bar{\Omega} ; \mathbb{R}_{\text {sym }}^{d \times d}\right)\right) .
\end{aligned}
$$

From our hypothesis, all the initial energies are uniformly bounded by a positive constant $\bar{E}$ independent of $n$; specifically,

$$
\int_{\Omega}\left[\frac{1}{2} \frac{\left|\mathbf{m}_{0, n}\right|^{2}}{\varrho_{0, n}}+P\left(\varrho_{0, n}\right)\right] d x \leq \bar{E}
$$

From (4.14) and the energy inequality (4.15) it is easy to deduce the following uniform bounds

$$
\begin{aligned}
& \operatorname{ess} \sup _{t>0}\left\|\frac{\mathbf{m}_{n}}{\sqrt{\varrho_{n}}}(t, \cdot)\right\|_{L^{2}\left(\Omega ; \mathbb{R}^{d}\right)} \leq c_{1}=c_{1}(\bar{E}), \\
& \operatorname{ess} \sup _{t>0}\left\|P\left(\varrho_{n}\right)(t, \cdot)\right\|_{L^{1}(\Omega)} \leq c(\bar{E}), \\
& \operatorname{ess}_{\sup } \sin _{t>0}\left\|\operatorname{Tr}\left[\Re_{n}(t)\right]\right\|_{\mathcal{M}^{+}(\bar{\Omega})} \leq c(\bar{E}), \\
& \left\|F\left(\mathbb{D} \mathbf{u}_{n}\right)\right\|_{L^{1}((0, \infty) \times \Omega)} \leq c(\bar{E}), \\
& \left\|F^{*}\left(\mathbb{S}_{n}\right)\right\|_{L^{1}((0, \infty) \times \Omega)} \leq c(\bar{E}) .
\end{aligned}
$$

5.1.1. Convergences of $\varrho_{n}$ and $\mathbf{m}_{n}$. For $\gamma>1$, from (4.8) and (5.10) we can easily deduce, passing to a suitable subsequence as the case may be,

$$
\varrho_{n} \stackrel{*}{\rightarrow} \varrho \quad \text { in } L^{\infty}\left(0, \infty ; L^{\gamma}(\Omega)\right) .
$$

Similarly, from (5.14), (5.9) and the fact that for a.e. $t>0$

$$
\left\|\mathbf{m}_{n}(t, \cdot)\right\|_{L^{\frac{2 \gamma}{\gamma+1}\left(\Omega ; \mathbb{R}^{d}\right)}} \leq\left\|\frac{\mathbf{m}_{n}}{\sqrt{\varrho_{n}}}(t, \cdot)\right\|_{L^{2}\left(\Omega ; \mathbb{R}^{d}\right)}\left\|\sqrt{\varrho_{n}}(t, \cdot)\right\|_{L^{2 \gamma}(\Omega)} \leq c(\bar{E}),
$$


passing to a suitable subsequence, we obtain

$$
\mathbf{m}_{n} \stackrel{*}{\rightarrow} \mathbf{m} \quad \text { in } L^{\infty}\left(0, \infty ; L^{\frac{2 \gamma}{\gamma+1}}\left(\Omega ; \mathbb{R}^{d}\right)\right) .
$$

Since the $L^{1}$-space is not reflexive, for $\gamma=1$ a more detailed analysis is needed. If we consider the Young function $\Phi(z)=z \log ^{+} z$, the densities $\left\{\varrho_{n}\right\}_{n \in \mathbb{N}}$ can be seen as uniformly bounded in $L^{\infty}\left(0, \infty ; L_{\Phi}(\Omega)\right)$, where $L_{\Phi}(\Omega)$ is the Orlicz space associated to $\Phi$; indeed, noticing that

$$
\varrho \log ^{+} \varrho=\left\{\begin{array}{ll}
0 & \text { if } 0 \leq \varrho<1, \\
\varrho \log \varrho & \text { if } \varrho \geq 1,
\end{array} \text { and } \quad-\frac{1}{e} \leq \varrho \log \varrho \leq 0 \quad \text { if } 0 \leq \varrho \leq 1,\right.
$$

from (5.10), for a.e. $\tau>0$ we have

$$
\begin{aligned}
\int_{\Omega} \varrho \log ^{+} \varrho(\tau, \cdot) d x & =\int_{\{\varrho \geq 1\}} \varrho \log \varrho(\tau, \cdot) d x \\
& \leq \int_{\Omega} \varrho \log \varrho(\tau, \cdot) d x-\int_{\{0 \leq \varrho<1\}} \varrho \log \varrho(\tau, \cdot) d x \\
& \leq c(\bar{E})+\frac{1}{e}|\Omega| .
\end{aligned}
$$

As the function $\Phi$ satisfies the $\Delta_{2}$-condition, $L_{\Phi}(\Omega)$ can be seen as the dual space of the Orlicz space $L_{\Psi}(\Omega)$, where $\Psi$ denotes the complementary Young function of $\Phi$, and hence, passing to a suitable subsequence, we get

$$
\varrho_{n} \stackrel{*}{\rightarrow} \varrho \quad \text { in } L^{\infty}\left(0, \infty ; L_{\Phi}(\Omega)\right) .
$$

We are now able to prove the uniform integrability of the sequence $\left\{\mathbf{m}_{n}(t, \cdot)\right\}_{n \in \mathbb{N}} \subset L^{1}\left(\Omega ; \mathbb{R}^{d}\right)$ for a.e. $t>0$. More precisely, we want to show that for every $\varepsilon>0$ there exists $\delta=\delta(\varepsilon)$ such that for all $n \in \mathbb{N}$ and a.e. $t>0$

$$
\int_{M}\left|\mathbf{m}_{n}\right|(t, \cdot) d x<\varepsilon \quad \text { for all } M \subset \Omega \text { such that }|M|<\delta .
$$

Fix $\varepsilon>0$ and choose $\tilde{\varepsilon}=\tilde{\varepsilon}(\varepsilon)$ such that $\tilde{\varepsilon}=\left(\varepsilon / c_{1}\right)^{2}$, with $c_{1}$ as in (5.9). The superlinearity of $P(4.9)$ combined with the uniform bound (5.10) guarantees that the sequence $\left\{\varrho_{n}(t, \cdot)\right\}_{n \in \mathbb{N}} \subset L^{1}(\Omega)$ is uniformly integrable for a.e. $t>0$, as a consequence of de la Vallée-Poussin criterion; thus, there exists $\delta=\delta(\tilde{\varepsilon})$ such that for all $n \in \mathbb{N}$ and a.e. $t>0$,

$$
\int_{M} \varrho_{n}(t, \cdot) d x<\tilde{\varepsilon} \text { for all } M \subset \Omega \text { such that }|M|<\delta .
$$

Fix $M \subset \Omega$ with $|M|<\delta$; applying Hölder's inequality, (5.9), (5.17) and writing

$$
\mathbf{m}=\sqrt{\varrho} \frac{\mathbf{m}}{\sqrt{\varrho}}
$$

we get that for all $n \in \mathbb{N}$ and a.e. $t>0$

$$
\int_{M}\left|\mathbf{m}_{n}\right|(t, \cdot) d x \leq\left(\int_{M} \varrho_{n}(t, \cdot) d x\right)^{\frac{1}{2}}\left(\int_{M} \frac{\left|\mathbf{m}_{n}\right|^{2}}{\varrho_{n}}(t, \cdot) d x\right)^{\frac{1}{2}}<c_{1} \tilde{\varepsilon}^{\frac{1}{2}}=\varepsilon .
$$

Dunford-Pettis theorem ensures that for a.e. $t>0$ the sequence $\left\{\mathbf{m}_{n}(t, \cdot)\right\}_{n \in \mathbb{N}} \subset L^{1}\left(\Omega ; \mathbb{R}^{d}\right)$ is relatively compact with respect to the weak topology; in particular, we have that

$$
\mathbf{m}_{n} \rightarrow \mathbf{m} \text { in } L_{\mathrm{loc}}^{1}\left(0, \infty ; L^{1}\left(\Omega ; \mathbb{R}^{d}\right)\right) .
$$

Next, to get (5.2) from (5.14) and (5.16) we have to show that the family of $t$-dependent functions

$$
f_{n}(t):=\int_{\Omega} \varrho_{n}(t, \cdot) \phi d x
$$


converges strongly in $C([a, b])$ for any $\phi \in C_{c}^{\infty}(\Omega)$ and any compact subset $[a, b] \subset(0, \infty)$. Recalling that the densities $\varrho_{n}$ and the momenta $\mathbf{m}_{n}$ are weakly continuous in time, the sequences $\left\{f_{n}\right\}_{n \in \mathbb{N}}$ and $\left\{f_{n}^{\prime}\right\}_{n \in \mathbb{N}}$ are uniformly bounded in $[a, b]$, since for all $\gamma \geq 1$

$$
\sup _{t \in[a, b]}\left|f_{n}(t)\right| \leq \sup _{t \in[a, b]}\left\|\varrho_{n}(t, \cdot)\right\|_{L^{\gamma}(\Omega)}\|\phi\|_{L^{\gamma^{\prime}}(\Omega)} \leq c(\phi),
$$

while from the uniform boundedness of the momenta $\mathbf{m}_{n}$ in $L^{\infty}\left(0, \infty ; L^{p}\left(\Omega ; \mathbb{R}^{d}\right)\right)$ with $p=\frac{2 \gamma}{\gamma+1}$ and $\gamma \geq 1$

$$
\sup _{t \in[a, b]}\left|f_{n}^{\prime}(t)\right| \leq \sup _{t \in[a, b]}\left\|\mathbf{m}_{n}(t, \cdot)\right\|_{L^{p}\left(\Omega: \mathbb{R}^{d}\right)}\left\|\nabla_{x} \phi\right\|_{L^{p^{\prime}}\left(\Omega ; \mathbb{R}^{d}\right)} \leq c(\phi) .
$$

As a consequence of the Arzelà-Ascoli theorem, we get (5.2). A similar argument can be applied to get (5.3).

Finally, notice that, from the Sobolev embedding (4.19), $\varrho_{n}:[0, \infty) \rightarrow W^{-k, 2}(\Omega)$ is a continuous function for all $n \in \mathbb{N}$, and thus by Proposition 2.1 showing

$$
\varrho_{n} \rightarrow \varrho \quad \text { in } \mathfrak{D}\left([0, \infty) ; W^{-k, 2}(\Omega)\right)
$$

is equivalent to

$$
\sup _{t \in[0, M]}\left|\int_{\Omega}\left(\varrho_{n}-\varrho\right)(t, \cdot) e_{k} d x\right| \rightarrow 0, \quad \text { for all } k, M \in \mathbb{N},
$$

where $\left\{e_{k}\right\}_{k \in \mathbb{N}}$ is an orthonormal basis of $L^{2}(\Omega)$; but this easily follows from convergence (5.2) and Parseval's identity. The same argument can be applied to show that

$$
\mathbf{m}_{n} \rightarrow \mathbf{m} \quad \text { in } \mathfrak{D}\left([0, \infty) ; W^{-k, 2}\left(\Omega ; \mathbb{R}^{d}\right)\right) .
$$

5.1.2. Convergences of $\mathbf{u}_{n}$ and $\mathbb{S}_{n}$. From (4.6) and (5.12) we can also deduce that

$$
\left\|\mathbb{D} \mathbf{u}_{n}-\frac{1}{d}\left(\operatorname{div}_{x} \mathbf{u}_{n}\right) \mathbb{I}\right\|_{L^{q}\left((0, \infty) \times \Omega ; \mathbb{R}^{d \times d}\right)} \leq c(\bar{E}) .
$$

Fixing a compact interval $[a, b] \subset(0,+\infty)$ and an open bounded interval $I$ such that $[a, b] \subset I$, the previous inequality combined with the $L^{q}$-version of the trace-free Korn's inequality (see for instance [6], Theorem 3.1) implies

$$
\left\|\nabla_{x} \mathbf{u}_{n}\right\|_{L^{q}\left(I \times \Omega ; \mathbb{R}^{d \times d}\right)} \leq c(\bar{E}) ;
$$

the standard Poincaré inequality ensures then

$$
\left\|\mathbf{u}_{n}\right\|_{L^{q}\left(I ; W_{0}^{1, q}\left(\Omega ; \mathbb{R}^{d}\right)\right)} \leq c(\bar{E})
$$

and thus we get convergence (5.4).

The superlinearity of $F^{*}$ (4.5) combined with (5.13), the de la Vallée-Poussin criterion and the Dunford-Pettis theorem, gives convergence (5.5).

5.1.3. Convergences of $\boldsymbol{p}\left(\varrho_{n}\right), \frac{\mathbf{m}_{n} \otimes \mathbf{m}_{n}}{\varrho_{n}}$ and $\mathfrak{R}_{n}$. Notice that in (5.7) we don't consider the case $\gamma=1$ because it reduces to (5.2). On the other side, when $\gamma>1$, estimates (5.9) and (5.10), combined with the fact that

$$
\left|\frac{\mathbf{m} \otimes \mathbf{m}}{\varrho}\right| \leq c \frac{|\mathbf{m}|^{2}}{\varrho}, \quad p(\varrho) \leq c(1+P(\varrho))
$$

for some positive constants $c$, imply that the pressures $\left\{p\left(\varrho_{n}(t, \cdot)\right)\right\}_{n \in \mathbb{N}}$ and the convective terms $\left\{\frac{\mathbf{m}_{n} \otimes \mathbf{m}_{n}}{\varrho_{n}}(t, \cdot)\right\}_{n \in \mathbb{N}}$ are uniformly bounded in the non-reflexive $L^{1}$-space for a.e. $t>0$. The idea is then to see the $L^{1}$-space as embedded in the space of bounded Radon measures $\mathcal{M}(\bar{\Omega})$, which in turn can be identified as the dual space of the separable space $C(\bar{\Omega})$. Accordingly, introducing the space $L_{\text {weak }}^{\infty}(0, \infty ; \mathcal{M}(\bar{\Omega}))$, cf. Remark 4.2, we obtain convergences (5.6) and (5.7). Finally, estimate (5.11) guarantees convergence $(5.8)$. 


\subsection{Limit Passage}

We are now ready to pass to the limit in the weak formulation of the continuity equation and the balance of momentum, obtaining that

$$
\left[\int_{\Omega} \varrho \varphi(t, \cdot) d x\right]_{t=0}^{t=\tau}=\int_{0}^{\tau} \int_{\Omega}\left[\varrho \partial_{t} \varphi+\mathbf{m} \cdot \nabla_{x} \varphi\right] d x d t
$$

holds $\tau>0$ and any $\varphi \in C_{c}^{1}([0, \infty) \times \bar{\Omega})$, with $\varrho(0, \cdot)=\varrho_{0}$, and

$$
\begin{aligned}
& {\left[\int_{\Omega} \mathbf{m} \cdot \boldsymbol{\varphi}(t, \cdot) d x\right]_{t=0}^{t=\tau}=\int_{0}^{\tau} \int_{\Omega}\left[\mathbf{m} \cdot \partial_{t} \boldsymbol{\varphi}+\overline{\mathbb{1}_{\varrho>0} \frac{\mathbf{m} \otimes \mathbf{m}}{\varrho}}: \nabla_{x} \boldsymbol{\varphi}+\overline{p(\varrho)} \operatorname{div}_{x} \boldsymbol{\varphi}\right] d x d t} \\
& -\int_{0}^{\tau} \int_{\Omega} \mathbb{S}: \nabla_{x} \varphi d x d t+\int_{0}^{\tau} \int_{\bar{\Omega}} \nabla_{x} \varphi: d \widetilde{\Re} d t
\end{aligned}
$$

holds for any $\tau>0$ and any $\varphi \in C_{c}^{1}\left([0, \infty) \times \bar{\Omega} ; \mathbb{R}^{d}\right),\left.\varphi\right|_{\partial \Omega}=0$ with $\mathbf{m}(0, \cdot)=\mathbf{m}_{0}$. The last integral identity can be rewritten as

$$
\begin{aligned}
{\left[\int_{\Omega} \mathbf{m} \cdot \boldsymbol{\varphi}(t, \cdot) d x\right]_{t=0}^{t=\tau} } & =\int_{0}^{\tau} \int_{\Omega}\left[\mathbf{m} \cdot \partial_{t} \boldsymbol{\varphi}+\mathbb{1}_{\varrho>0} \frac{\mathbf{m} \otimes \mathbf{m}}{\varrho}: \nabla_{x} \boldsymbol{\varphi}+p(\varrho) \operatorname{div} x \boldsymbol{\varphi}\right] d x d t \\
& -\int_{0}^{\tau} \int_{\Omega} \mathbb{S}: \nabla_{x} \varphi d x d t+\int_{0}^{\tau} \int_{\bar{\Omega}} \nabla_{x} \boldsymbol{\varphi}: d \check{\Re} d t
\end{aligned}
$$

where $\check{\mathfrak{R}} \in L_{\text {weak }}^{\infty}\left(0, \infty ; \mathcal{M}\left(\bar{\Omega} ; \mathbb{R}_{\text {sym }}^{d \times d}\right)\right)$ is such that

$$
d \check{\mathfrak{R}}=d \widetilde{\mathfrak{R}}+\left(\overline{\mathbb{1}_{\varrho>0} \frac{\mathbf{m} \otimes \mathbf{m}}{\varrho}}-\mathbb{1}_{\varrho>0} \frac{\mathbf{m} \otimes \mathbf{m}}{\varrho}\right) d x+(\overline{p(\varrho)}-p(\varrho)) \chi(\gamma) \mathbb{I} d x,
$$

with

$$
\chi(\gamma)= \begin{cases}0 & \text { if } \gamma=1 \\ 1 & \text { if } \gamma>1\end{cases}
$$

We can prove the stronger condition

$$
\check{\mathfrak{R}} \in L_{\text {weak }}^{\infty}\left(0, \infty ; \mathcal{M}^{+}\left(\bar{\Omega} ; \mathbb{R}_{\text {sym }}^{d \times d}\right)\right) ;
$$

more precisely, we want to show that for all $\xi \in \mathbb{R}^{d}$, all open sets $\mathcal{B} \subset \Omega$ and a.e. $\tau>0$

$$
\check{\mathfrak{R}}(\tau):(\xi \otimes \xi)(\mathcal{B}) \geq 0 .
$$

we can rewrite the term on the left-hand side as

$$
\begin{aligned}
\int_{\mathcal{B}}(\xi \otimes \xi): d \check{\mathfrak{R}}(\tau+) & =\lim _{d \rightarrow 0} \int_{\tau}^{\tau+d} \int_{\mathcal{B}}(\xi \otimes \xi): d \check{\mathfrak{R}}(t) d t \\
& =\lim _{d \rightarrow 0} \int_{0}^{\infty} \int_{\bar{\Omega}} \mathbb{1}_{[\tau, \tau+d] \times \mathcal{B}}(\xi \otimes \xi): d \check{\mathfrak{R}}(t) d t
\end{aligned}
$$

Since the indicator function $\mathbb{1}_{[\tau-d, \tau+d] \times \mathcal{B}}$ can be approximated by some non-negative test functions, it is enough to show that

$$
\int_{0}^{\infty} \int_{\bar{\Omega}} \varphi(\xi \otimes \xi): d \check{\mathfrak{R}}(t) d t \geq 0
$$

holds for all $\varphi \in C_{c}^{\infty}((0, T) \times \Omega), \varphi \geq 0$. We can notice that the first term on the right-hand side of (5.18) will obviously satisfy the above inequality since $\widetilde{\Re}$ belongs to $L_{\text {weak }}^{\infty}\left(0, \infty ; \mathcal{M}^{+}\left(\bar{\Omega} ; \mathbb{R}_{\text {sym }}^{d \times d}\right)\right)$, and

$$
\int_{0}^{\infty} \int_{\bar{\Omega}}(\overline{p(\varrho)}-p(\varrho)) \mathbb{I}:(\xi \otimes \xi) \varphi d x d t=\int_{0}^{\infty} \int_{\bar{\Omega}}(\overline{p(\varrho)}-p(\varrho))|\xi|^{2} \varphi d x d t \geq 0,
$$


since $\varrho \mapsto p(\varrho)$ is convex and weakly lower semi-continuous in $L^{1}$, which implies $\overline{p(\varrho)} \geq p(\varrho)$, see for instance [14], Theorem 2.11. Finally, following the same idea developed in [15], Section 3.2, as a consequence of (5.6) we can write

$$
\begin{aligned}
\int_{0}^{\infty} \int_{\bar{\Omega}}\left(\overline{\mathbb{1}_{\varrho>0} \frac{\mathbf{m} \otimes \mathbf{m}}{\varrho}}\right. & \left.-\mathbb{1}_{\varrho>0} \frac{\mathbf{m} \otimes \mathbf{m}}{\varrho}\right):(\xi \otimes \xi) \varphi d x d t \\
& =\lim _{n \rightarrow \infty} \int_{0}^{\infty} \int_{\bar{\Omega}}\left(\mathbb{1}_{\varrho_{n}>0} \frac{\mathbf{m}_{n} \otimes \mathbf{m}_{n}}{\varrho_{n}}-\mathbb{1}_{\varrho>0} \frac{\mathbf{m} \otimes \mathbf{m}}{\varrho}\right):(\xi \otimes \xi) \varphi d x d t \\
& =\lim _{n \rightarrow \infty} \int_{0}^{\infty} \int_{\bar{\Omega}}\left(\mathbb{1}_{\varrho_{n}>0} \frac{\left|\mathbf{m}_{n} \cdot \xi\right|^{2}}{\varrho_{n}}-\mathbb{1}_{\varrho>0} \frac{|\mathbf{m} \cdot \xi|^{2}}{\varrho}\right) \varphi d x d t .
\end{aligned}
$$

The Cauchy-Schwarz inequality allows to write $|\mathbf{m} \cdot \xi|^{2} \leq|\mathbf{m}|^{2}|\xi|^{2}$, and thus by (5.9) we obtain

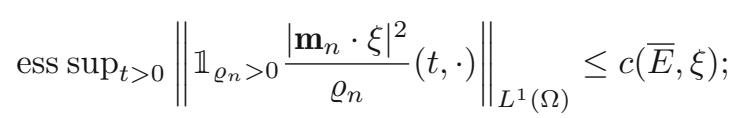

it is possible then to find the limit

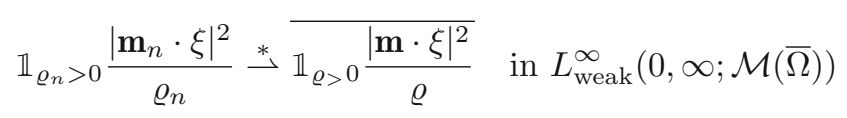

and rewrite the first line in (5.20) as

$$
\int_{0}^{\infty} \int_{\bar{\Omega}}\left(\overline{\mathbb{1}_{\varrho>0} \frac{|\mathbf{m} \cdot \xi|^{2}}{\varrho}}-\mathbb{1}_{\varrho>0} \frac{|\mathbf{m} \cdot \xi|^{2}}{\varrho}\right) \varphi d x d t .
$$

as in the previous passage, (5.19) will now follow from the weak lower semi-continuity on $D$ of the convex function $[\varrho, \mathbf{m}] \mapsto \frac{|\mathbf{m} \cdot \xi|^{2}}{\varrho}$. We proved in particular that the pair of functions $[\varrho, \mathbf{m}]$ satisfies conditions (ii) and (iii) of Definition 4.1. However, $\check{\mathfrak{R}}$ has to be slightly modified in order to get the energy (4.14), as we will see in Sect. 5.4.

\subsection{Auxiliary Lemma}

In order to prove that

$$
\mathbf{m}=\varrho \mathbf{u} \text { a.e. in }(0, \infty) \times \Omega,
$$

and in particular to show that $[\varrho, \mathbf{m}]$ satisfy condition (iv) of Definition 4.1, we need the following result.

Lemma 5.2. Let $\Omega \subset \mathbb{R}^{d}$ be a bounded domain. Suppose

$$
\left\{\varrho_{n}\right\}_{n \in \mathbb{N}} \text { to be uniformly bounded in } L^{\infty}\left(0, \infty ; L_{\Phi}(\Omega)\right),
$$

where $L_{\Phi}(\Omega)$ is the Orlicz space associated to the Young function $\Phi$ satisfying the $\Delta_{2}$-condition, with $\varrho_{n} \geq 0$ for all $n \in \mathbb{N}$. Suppose also that

$$
\partial_{t} \varrho_{n}=-\operatorname{div}_{x} \boldsymbol{g}_{n}
$$

where

$$
\left\{\boldsymbol{g}_{n}\right\}_{n \in \mathbb{N}} \text { is uniformly bounded in } L^{\infty}\left(0, \infty ; L^{p}\left(\Omega ; \mathbb{R}^{d}\right)\right)
$$

for some $p \geq 1$. Moreover, let

$$
\left\{\boldsymbol{u}_{n}\right\}_{n \in \mathbb{N}} \text { be uniformly bounded in } L_{\text {loc }}^{q}\left(0, \infty ; W^{1, q}\left(\Omega ; \mathbb{R}^{d}\right)\right), q>1 \text {. }
$$

Finally, let the sequence $\left\{\varrho_{n} \boldsymbol{u}_{n}\right\}_{n \in \mathbb{N}}$ be equi-integrable in $L_{\mathrm{loc}}^{1}\left(0, \infty ; L^{1}\left(\Omega ; \mathbb{R}^{d}\right)\right)$. 
Then, if

$$
\begin{aligned}
& \varrho_{n} \rightarrow \varrho \quad \text { in } C_{\text {weak }, \mathrm{loc}}\left([0, \infty) ; L^{1}(\Omega)\right), \\
& \boldsymbol{u}_{n} \rightarrow \boldsymbol{u} \quad \text { in } L_{\mathrm{loc}}^{q}\left(0, \infty ; W^{1, q}\left(\Omega ; \mathbb{R}^{d}\right)\right),
\end{aligned}
$$

and

$$
\varrho_{n} \boldsymbol{u}_{n} \rightarrow \boldsymbol{m} \quad \text { in } L_{\mathrm{loc}}^{1}\left(0, \infty ; L^{1}\left(\Omega ; \mathbb{R}^{d}\right)\right),
$$

we have

$$
\boldsymbol{m}=\varrho \boldsymbol{u} \quad \text { a.e. in }(0, \infty) \times \Omega .
$$

Proof. 1. Truncation. Following the same strategy developed in [1], Lemma 8.1, it is enough to suppose that

$$
\left\{\mathbf{u}_{n}\right\}_{n \in \mathbb{N}} \text { is uniformly bounded in } L_{\mathrm{loc}}^{\infty}\left(0, \infty ; L^{\infty}\left(\Omega ; \mathbb{R}^{d}\right)\right) .
$$

Considering the truncation $T_{k}\left(\mathbf{u}_{n}\right)$, notice that in this case we have

$$
\int_{a}^{b} \int_{\Omega} \varrho_{n}\left|\mathbf{u}_{n}-T_{k}\left(\mathbf{u}_{n}\right)\right| d x d t \leq 2 \iint_{\left\{\left|\mathbf{u}_{n}\right| \geq k\right\}} \varrho_{n}\left|\mathbf{u}_{n}\right| d x d t \rightarrow 0 \quad \text { as } k \rightarrow \infty, \text { uniformly in } n,
$$

for every $[a, b] \subset(0, \infty)$, in view of the equi-integrability of $\left\{\varrho_{n} \mathbf{u}_{n}\right\}_{n \in \mathbb{N}}$.

2. Regularization. We claim that it is sufficient to suppose

$$
\left\{\mathbf{u}_{n}\right\}_{n \in \mathbb{N}} \text { to be uniformly bounded in } L_{\text {loc }}^{q}\left(0, \infty ; W^{m, r}\left(\Omega ; \mathbb{R}^{d}\right)\right)
$$

with $q>1$ and $m, r$ arbitrarily large. Seeing all the quantities involved as embedded in $\mathbb{R}^{d}$ with compact support, we consider regularization in the spatial variable by convolution with a family of regularizing kernels $\left\{\theta_{\delta}\right\}_{\delta>0}$,

$$
\theta_{\delta}(x)=\frac{1}{\delta^{d}} \theta\left(\frac{x}{\delta}\right),
$$

where $\theta$ is a bell-shaped function such that

$$
\theta \in C_{c}^{\infty}\left(\mathbb{R}^{d}\right), \quad \theta \geq 0, \quad \theta(x)=\theta(|x|), \quad \int_{\mathbb{R}^{d}} \theta(x) d x=1 .
$$

As in the previous step, writing

$$
\varrho_{n} \mathbf{u}_{n}=\varrho_{n} \theta_{\delta} * \mathbf{u}_{n}+\varrho_{n}\left(\mathbf{u}_{n}-\theta_{\delta} * \mathbf{u}_{n}\right),
$$

our goal is tho show that for every $[a, b] \subset(0, \infty)$

$$
\int_{a}^{b} \int_{\Omega} \varrho_{n}\left|\mathbf{u}_{n}-\theta_{\delta} * \mathbf{u}_{n}\right| d x d t \rightarrow 0 \quad \text { as } \delta \rightarrow 0, \text { uniformly in } n .
$$

To this end, we introduce the Banach space

$$
X=W_{0}^{1, q} \cap L^{\infty}\left(\Omega ; \mathbb{R}^{d}\right)
$$

and observe that, in view of (5.23) and (5.26),

$$
\left\{\left\|\mathbf{u}_{n}\right\|_{X}\right\}_{n \in \mathbb{N}} \text { is uniformly bounded in } L_{\text {loc }}^{q}(0, \infty) .
$$

Consequently, we may write

$$
\int_{a}^{b} \int_{\Omega} \varrho_{n}\left|\mathbf{u}_{n}-\theta_{\delta} * \mathbf{u}_{n}\right| d x d t=I_{1}^{M}+I_{2}^{M}
$$


with

$$
\begin{aligned}
& I_{1}^{M}=\int_{\left\{\left\|\mathbf{u}_{n}(t, \cdot)\right\|_{X} \leq M\right\}} \int_{\Omega} \varrho_{n}\left|\mathbf{u}_{n}-\theta_{\delta} * \mathbf{u}_{n}\right| d x d t, \\
& I_{2}^{M}=\int_{\left\{\left\|\mathbf{u}_{n}(t, \cdot)\right\|_{X}>M\right\}} \int_{\Omega} \varrho_{n}\left|\mathbf{u}_{n}-\theta_{\delta} * \mathbf{u}_{n}\right| d x d t,
\end{aligned}
$$

where, in view of (5.28) - recall that the functions $\varrho_{n}$ are weakly continuous in time

$$
I_{2}^{M} \leq c \sup _{t \in[a, b]}\left\|\varrho_{n}(t, \cdot)\right\|_{L^{1}(\Omega)}\left\|\mathbf{u}_{n}\right\|_{L^{\infty}\left((a, b) \times \Omega ; \mathbb{R}^{d}\right)}\left|\left\{\left\|\mathbf{u}_{n}(t, \cdot)\right\|_{X}>M\right\}\right| \rightarrow 0 \quad \text { as } M \rightarrow \infty,
$$

uniformly in $n$ and independently of $\delta$.

It remains to show smallness of the first integral for fixed $M$. To this end, denoting with $\Psi$ the complementary Young function of $\Phi$, we consider the Orlicz space $L_{\Psi}(\Omega)$ that can be identified with the dual of $L_{\Phi}(\Omega)$ as $\Phi$ satisfies the $\Delta_{2}$-condition. By Proposition 5.3 below, we recover the compact embedding

$$
X \hookrightarrow \hookrightarrow L_{\Psi}\left(\Omega ; \mathbb{R}^{d}\right)
$$

which, combined with boundedness of convolution on $L_{\Psi}(\Omega)$ (see [19], Lemma 4.4.3), gives

$$
I_{1}^{M} \leq \sup _{t \in[a, b]}\left\|\varrho_{n}(t, \cdot)\right\|_{L_{\Phi}(\Omega)} \sup _{\|\mathbf{u}\|_{X} \leq M}\left\|\mathbf{u}-\theta_{\delta} * \mathbf{u}\right\|_{L_{\Psi}\left(\Omega ; \mathbb{R}^{d}\right)} \rightarrow 0 \quad \text { as } \delta \rightarrow 0 .
$$

3. Conclusion. Using the Sobolev embedding

$$
L^{1}(\Omega) \hookrightarrow \hookrightarrow W^{-1, s^{\prime}} \quad \text { for any } s>d,
$$

from (5.24) we get that

$$
\varrho_{n} \rightarrow \varrho \quad \text { in } C_{\mathrm{loc}}\left([0, \infty) ; W^{-1, s^{\prime}}(\Omega)\right) \text { for any } s>d,
$$

and thus, to conclude the proof of the Lemma it is sufficient to choose $m=1$ and $r=s$ in (5.27).

Proposition 5.3. Let $\Omega \subset \mathbb{R}^{d}$ be a bounded domain. Then, for a fixed $q \geq 1$

$$
X=W_{0}^{1, q} \cap L^{\infty}(\Omega) \hookrightarrow \hookrightarrow L_{\Phi}(\Omega),
$$

where $L_{\Phi}$ is the Orlicz space associated to the Young function $\Phi$.

Proof. Let $K$ be a bounded set of $X$ and let $\Phi_{1}$ be a Young function such that $\Phi \prec \prec \Phi_{1}$, i.e.

$$
\lim _{t \rightarrow \infty} \frac{\Phi(t)}{\Phi_{1}(\lambda t)}=0
$$

for all $\lambda>0$. Then, in particular, $K$ is bounded in the Orlicz space $L_{\Phi_{1}}(\Omega)$; indeed, denoting with $\Psi$ the complementary Young function of $\Phi$, we have that for every $u \in K$ and every $v$ belonging to the Orlicz class $\widetilde{L}_{\Psi}(\Omega)$

$$
\int_{\Omega}|u(x) v(x)| d x \leq\|u\|_{L^{\infty}(\Omega)}\|v\|_{L^{1}(\Omega)} \leq\|u\|_{L^{\infty}(\Omega)} \sigma(v ; \Psi)
$$

and thus

$$
\|u\|_{L_{\Phi_{1}}(\Omega)}=\sup _{\substack{v \in \widetilde{L}_{\Psi}(\Omega) \\ \sigma(v ; \Psi) \leq 1}} \int_{\Omega}|u(x) v(x)| d x \leq\|u\|_{L^{\infty}(\Omega)} \leq\|u\|_{X} \leq c,
$$

where $\|u\|_{X}=\max \left\{\|u\|_{W^{1, q}(\Omega)},\|u\|_{L^{\infty}(\Omega)}\right\}$ and the constant $c$ is independent of the choice $u \in K$. Applying [22], Theorems 3.17.7 and 3.17.8 we get that

$$
L_{\Phi_{1}}(\Omega) \hookrightarrow E_{\Phi}(\Omega),
$$


where $E_{\Phi}(\Omega)$ is the closure of the set of all bounded measurable functions defined on $\Omega$ with respect to the Orlicz norm $\|\cdot\|_{L_{\Phi}}$, and that the functions in $K$ have uniformly continuous $L_{\Phi}$-norms, i.e., for every $\varepsilon>0$ there exists a $\delta=\delta(\varepsilon)>0$ such that

$$
\left\|u \mathbb{1}_{M}\right\|_{L_{\Phi}(\Omega)}<\varepsilon
$$

provided $M \in \Omega$ is measurable, $|M|<\delta$ and $u \in K$.

Furthermore, since

$$
W^{1, q}(\Omega) \hookrightarrow \hookrightarrow L^{1}(\Omega),
$$

the set $K$ is relatively compact in $L^{1}(\Omega)$ and consequently it is relatively compact with respect to the convergence in measure.

Finally, it is sufficient to apply [22], Theorem 3.14.11, which we report for reader's convenience.

Theorem 5.4. Let $K$ be a subset of $E_{\Phi}(\Omega)$ which is relatively compact in the sense of convergence in measure and such that the functions in $K$ have uniformly continuous $L_{\Phi}$-norms. Then $K$ is relatively compact in $L_{\Phi}$.

\subsection{Limit of the Energies}

From (4.14) we can notice that the energies $E_{n}(\tau)$ are non-increasing and for $\gamma>1$ they are also nonnegative, while for $\gamma=1$ we have

$$
\begin{aligned}
E_{n}(\tau) & \geq \int_{\Omega}\left[\frac{1}{2} \frac{\left|\mathbf{m}_{n}\right|^{2}}{\varrho_{n}}+\mathbb{1}_{\varrho_{n} \geq 1} \varrho \log \varrho\right](\tau, \cdot) d x+\frac{1}{\lambda_{n}} \int_{\bar{\Omega}} d \operatorname{Tr}\left[\Re_{n}(\tau)\right]+\int_{\left\{0 \leq \varrho_{n}<1\right\}} \varrho_{n} \log \varrho_{n} d x \\
& \geq \text { "non-negative term" }-\frac{|\Omega|}{e} .
\end{aligned}
$$

for a.e. $\tau>0$. Hence, for every $[a, b] \subset(0, \infty)$ and every $n \in \mathbb{N}$

$$
\begin{gathered}
\left\|E_{n}\right\|_{L^{1}[a, b]} \leq \int_{0}^{b}\left|E_{n}(t)\right| d t \leq b \sup _{t \in[0, b]}\left|E_{n}(t)\right| \leq b E_{0, n} \leq c(\bar{E}), \\
V_{a}^{b}\left(E_{n}\right)=\int_{a}^{b}\left|\frac{d}{d t} E_{n}\right|=-\int_{a}^{b} \frac{d}{d t} E_{n} d t=E_{n}(a)-E_{n}(b) \leq\left\{\begin{array}{ll}
E_{0, n}+\frac{|\Omega|}{e} & \text { if } \gamma=1 \\
E_{0, n} & \text { if } \gamma>1
\end{array} \leq c(\bar{E}),\right.
\end{gathered}
$$

so that $\left\{E_{n}\right\}_{n \in \mathbb{N}}$ is locally of bounded variation. We can then use Helly's selection theorem (compactness theorem for $B V_{\text {loc }}$ ): a sequence of functions that is locally of total bounded variation and uniformly bounded at a point has a convergent subsequence, pointwise and in $L_{\mathrm{loc}}^{1}$. Passing to a suitable subsequence as the case may be, we obtain

$$
E_{n}(t) \rightarrow E(t) \text { for every } t \in[0, \infty) \text { and in } L_{\mathrm{loc}}^{1}(0, \infty),
$$

which in particular implies

$$
E_{n} \rightarrow E \quad \text { in } \mathfrak{D}([0, \infty) ; \mathbb{R}),
$$

since $E_{n}:[0, \infty) \rightarrow \mathbb{R}$ is a monotone function for all $n \in \mathbb{N}$ and thus, by Proposition 2.1, showing (5.30) is equivalent to show almost everywhere convergence. 
On the other side, from (5.9), (5.10) and (5.11) we get

$$
\begin{aligned}
& \frac{\left|\mathbf{m}_{n}\right|^{2}}{\varrho_{n}} * \frac{\overline{|\mathbf{m}|^{2}}}{\varrho} \quad \text { in } L_{\text {weak }}^{\infty}(0, \infty ; \mathcal{M}(\bar{\Omega})) \\
& P\left(\varrho_{n}\right) \stackrel{*}{\rightarrow} \overline{P(\varrho)} \quad \text { in } L_{\text {weak }}^{\infty}(0, \infty ; \mathcal{M}(\bar{\Omega})) \\
& \frac{1}{\lambda_{n}} \operatorname{Tr}\left[\Re_{n}\right] \stackrel{*}{\rightarrow} \widetilde{\mathfrak{E}} \quad \text { in } L_{\mathrm{weak}}^{\infty}\left(0, \infty ; \mathcal{M}^{+}(\bar{\Omega})\right) \text {. }
\end{aligned}
$$

We can then write

$$
E(\tau)=\int_{\Omega}\left[\frac{1}{2} \frac{|\mathbf{m}|^{2}}{\varrho}+P(\varrho)\right](\tau, \cdot) d x+\int_{\bar{\Omega}} d \mathfrak{E}(\tau)
$$

for a.e. $\tau>0$, with

$$
d \mathfrak{E}=d \widetilde{\mathfrak{E}}+\frac{1}{2}\left(\frac{\overline{|\mathbf{m}|^{2}}}{\varrho}-\frac{|\mathbf{m}|^{2}}{\varrho}\right) d x+(\overline{P(\varrho)}-P(\varrho)) d x
$$

where, once again, from the convexity of the function $P$ and of the superposition $[\varrho, \mathbf{m}] \mapsto \frac{|\mathbf{m}|^{2}}{\varrho}$, we get

$$
\mathfrak{E} \in L_{\text {weak }}^{\infty}\left(0, \infty ; \mathcal{M}^{+}(\bar{\Omega})\right)
$$

As pointed out in Sect. 4.1.1, we can choose constant $\lambda>0$ such that

$$
\operatorname{Tr}[\check{\mathfrak{R}}(\tau)] \leq \lambda \mathfrak{E}(\tau)
$$

for a.e. $\tau \in(0, T)$; however, with this choice we only get

$$
\int_{\Omega}\left[\frac{1}{2} \frac{|\mathbf{m}|^{2}}{\varrho}+P(\varrho)\right](\tau, \cdot) \mathrm{d} x+\frac{1}{\lambda} \int_{\bar{\Omega}} \mathrm{d} \operatorname{Tr}[\check{\mathfrak{R}}(\tau)] \leq \mathrm{E}(\tau)
$$

for a.e. $\tau \in(0, T)$. To obtain (4.14), it is sufficient to define a new defect

$$
\mathfrak{R}=\check{\mathfrak{R}}+\psi(t) \mathbb{I},
$$

where the function $\psi \geq 0$ of time only can be chosen in such a way that

$$
\int_{\Omega}\left[\frac{1}{2} \frac{|\mathbf{m}|^{2}}{\varrho}+P(\varrho)\right](\tau, \cdot) \mathrm{d} x+\frac{1}{\lambda} \int_{\bar{\Omega}} \mathrm{d} \operatorname{Tr}[\Re(\tau)]=\mathrm{E}(\tau)
$$

for a.e. $\tau \in(0, T)$. Clearly,

$$
\int_{\bar{\Omega}} \nabla_{x} \varphi: d \Re=\int_{\bar{\Omega}} \nabla_{x} \varphi: d \check{\Re}
$$

for any $\varphi \in C_{c}^{\infty}\left([0, \infty) \times \bar{\Omega} ; \mathbb{R}^{d}\right),\left.\varphi\right|_{\partial \Omega}=0$, and therefore, the weak formulation of the balance of momentum (4.13) remains valid.

Finally, notice that the couple $[\varrho, \mathbf{m}]$ satisfies the energy inequality (4.15) due to lower semi-continuity of the functions $F$ and $F^{*}$ : for a.e. $\tau>0$

$$
\int_{0}^{\tau} \int_{\Omega}\left[F(\mathbb{D} \mathbf{u})+F^{*}(\mathbb{S})\right] d x d t \leq \liminf _{n \rightarrow \infty} \int_{0}^{\tau} \int_{\Omega}\left[F\left(\mathbb{D} \mathbf{u}_{n}\right)+F^{*}\left(\mathbb{S}_{n}\right)\right] d x d t
$$

in particular, $[\varrho, \mathbf{m}]$ satisfies condition (iv) of Definition 4.1.

Acknowledgements. This work was supported by the Einstein Foundation, Berlin. The author wishes to thank Prof. Eduard Feireisl for the helpful advice and discussions.

Funding Open Access funding provided by Projekt DEAL.

Compliance with ethical standards

Conflict of interest The author declares that there is no conflict of interest. 
Open Access. This article is licensed under a Creative Commons Attribution 4.0 International License, which permits use, sharing, adaptation, distribution and reproduction in any medium or format, as long as you give appropriate credit to the original author(s) and the source, provide a link to the Creative Commons licence, and indicate if changes were made. The images or other third party material in this article are included in the article's Creative Commons licence, unless indicated otherwise in a credit line to the material. If material is not included in the article's Creative Commons licence and your intended use is not permitted by statutory regulation or exceeds the permitted use, you will need to obtain permission directly from the copyright holder. To view a copy of this licence, visit http://creativecommons.org/licenses/by/4.0/.

Publisher's Note Springer Nature remains neutral with regard to jurisdictional claims in published maps and institutional affiliations.

\section{References}

[1] Abbatiello, A., Feireisl, E.: On a class of generalized solutions to equations describing incompressible viscous fluids. Annali di Matematica Pura e Applicata (1923); 2019

[2] Abbatiello, A., Feireisl, E., Novotný, A.: Generalized solutions to mathematical models of compressible viscous fluids, arXiv:1912.12896 (2019)

[3] Ambrosio, L.: Transport equation and Cauchy problem for BV vector fields. Inventiones Mathematicae 158, 227-260 (2004)

[4] Basarić, D.: Semiflow selection for the compressible Navier-Stokes system. J. Evol. Equ. (2020). https://doi.org/10. 1007/s00028-020-00578-x

[5] Basarić, D.: Vanishing viscosity limit for the compressible Navier-Stokes system via measure-valued solutions, arXiv:1903.05886 (2019)

[6] Breit, D., Cianchi, A., Diening, L.: Trace-free Korn inequality in Orlicz spaces. SIAM J. Math. Anal. 49(4), 2496-2516 (2017)

[7] Breit, D., Feireisl, E., Hofmanová, M.: Dissipative solutions and semiflow selection for the complete Euler system. Commun. Math. Phys. 376, 1471-1497 (2020). https://doi.org/10.1007/s00220-019-03662-7

[8] Breit, D., Feireisl, E., Hofmanová, M.: Markov selection for the stochastic compressible Navier-Stokes system, arXiv:1809.07265 (2018)

[9] Breit, D., Feireisl, E., Hofmanová, M.: Solution semiflow to the isentropic Euler system. Arch. Ration. Mech. Anal. 235, 167-194 (2020). https://doi.org/10.1007/s00205-019-01420-6

[10] Buckmaster, T., Vicol, V.: Convex integration and phenomenologies in turbulence, arXiv:1901.09023 (2019)

[11] Cardona, J.E., Kapitanski, L.: Semiflow selection and Markov selection theorems, arXiv:1707.04778 (2017)

[12] Crandall, M.G., Ishii, H., Lions, P.-L.: User's guide to viscosity solutions of second order partial differential equations. Bull. Am. Math. Soc. 27(1), 1-67 (1992)

[13] DiPerna, R.J., Lions, P.-L.: Ordinary differential equations, transport theory and Sobolev spaces. Inventiones Mathematicae 98, 511-547 (1989)

[14] Feireisl, E.: Dynamics of Viscous Compressible Fluids. Oxford University Press, Oxford (2003)

[15] Feireisl, E., Hofmanová, M.: On the vanishing viscosity limit of the isentropic Navier-Stokes system, arXiv:1905.02548 (2019)

[16] Feireisl, E., Kwon, Y.-S., Novotný, A.: On the long-time behaviour of dissipative solutions to models of non-Newtonian compressible fluids, arXiv:2001.03313 (2020)

[17] Feireisl, E., Novotný, A.: Stability of Planar Rarefaction Waves Under General Viscosity Perturbation of the Isentropic Euler System, Preprint No. 12-2020 of the Institute of Mathematics of the Czech Academy of Sciences (2020)

[18] Flandoli, F., Romito, M.: Markov selections for the 3D stochastics Navier-Stokes equations. Probab. Theory Rel. Fields 140(3-4), 407-458 (2008)

[19] Harjulehto, P., Hästö, P.: Orlicz Spaces and Generalized Orlicz Spaces. Lecture Notes in Mathematics, vol. 2236. Springer, Berlin (2019)

[20] Jakubowski, A.: On the Skorokhod topology. Annales de l'I. H. P. Sect. B 22(3), 263-285 (1986)

[21] Krylov, N.V.: The selection of a Markov process from a Markov system of processes, and the construction of quasidiffusion processes. Izv. Akad. Nauk SSSR Ser. Mat. 37, 691-708 (1973)

[22] Kufner, A., John, O., Fučík, S.: Function Spaces. Springer, Prague (1977)

[23] Measure-valued solution for non-Newtonian compressible isothermal monopolar fluid: Matušů-Nečasová, Š., Novotný, A. Acta Applicandae Mathematica 37, 109-128 (1994)

[24] Stroock, D.W., Varadhan, S.R.S.: Multidimensional Diffusion Processes, Classics in Mathematics. Springer, Berlin (2006)

[25] Whitt, W.: Stochastic-Process Limits: An Introduction to Stochastic-Process Limits and Their Applications to Queues, Springer Series In Operation Research (2002) 
Danica Basarić

Technische Universität Berlin Institute für Mathematik

Straße des 17 Juni 136

10623 Berlin

Germany

e-mail: basaric@math.tu-berlin.de

(accepted: August 21, 2020; published online: November 23, 2020) 